\title{
SENSITIVITY ANALYSIS FOR TWO-LEVEL VALUE FUNCTIONS WITH APPLICATIONS TO BILEVEL PROGRAMMING*
}

\author{
S. DEMPE $^{\dagger}$, B. S. MORDUKHOVICH ${ }^{\ddagger}$, AND A. B. ZEMKOHO ${ }^{\dagger}$
}

\begin{abstract}
This paper contributes to a deeper understanding of the link between a now conventional framework in hierarchical optimization called the optimistic bilevel problem and its initial more difficult formulation that we call here the original optimistic bilevel optimization problem. It follows from this research that although the process of deriving necessary optimality conditions for the latter problem is more involved, the conditions themselves do not - to a large extent- differ from those known for the conventional problem. It has already been well recognized in the literature that for optimality conditions of the usual optimistic bilevel program appropriate coderivative constructions for the set-valued solution map of the lower-level problem could be used, while it is shown in this paper that for the original optimistic formulation we have to go a step further to require and justify a certain Lipschitz-like property of this map. This is related to the local Lipschitz continuity of the optimal value function of an optimization problem constrained by solutions to another optimization problem; this function is labeled here as the two-level value function. More generally, we conduct a detailed sensitivity analysis for value functions of mathematical programs with extended complementarity constraints. The results obtained in this vein are applied to the two-level value function and then to the original optimistic formulation of the bilevel optimization problem, for which we derive verifiable stationarity conditions of various types entirely in terms of the initial data.
\end{abstract}

Key words. bilevel programming, coderivative, Lipschitz-like property, sensitivity analysis, two-level value function, MPCC value functions, optimality conditions

AMS subject classifications. 90C26, 90C30, 90C31, 90C46, 91C12, 91A65

DOI. $10.1137 / 110845197$

1. Introduction. This paper is mainly motivated by the study of a class of the so-called bilevel programming problems generally formalized as

$$
\text { "min" } \left.\min _{x}(x, y) \mid x \in X, y \in S(x)\right\},
$$

where $F: \mathbb{R}^{n} \times \mathbb{R}^{m} \rightarrow \mathbb{R}$ and $X \subset \mathbb{R}^{n}$ stands for the upper-level/leader's objective function and the feasible set, respectively, while the multifunction $S: \mathbb{R}^{n} \rightrightarrows \mathbb{R}^{m}$ denotes the set-valued solution/argminimum map for the lower-level/follower's problem

$$
\min _{y}\{f(x, y) \mid y \in K(x)\}
$$

with the lower-level objective function $f: \mathbb{R}^{n} \times \mathbb{R}^{m} \rightarrow \mathbb{R}$. For simplicity we confine ourselves to the case where the upper- and lower-level constraints are given by

$$
X:=\left\{x \in \mathbb{R}^{n} \mid G(x) \leq 0\right\} \text { and } K(x):=\left\{y \in \mathbb{R}^{m} \mid g(x, y) \leq 0\right\},
$$

*Received by the editors August 22, 2011; accepted for publication (in revised form) July 18, 2012; published electronically October 9, 2012.

http://www.siam.org/journals/siopt/22-4/84519.html

$\dagger$ Institut für Numerische Mathematik und Optimierung, Technische Universität Bergakademie Freiberg, Akademie Straße 6, 09596 Freiberg, Germany (dempe@math.tu-freiberg.de, zemkoho@ math.tu-freiberg.de). Part of the third author's work was completed while visiting the Department of Mathematics at Wayne State University, Detroit. The research of the third author was supported by the Deutscher Akademischer Austausch Dienst (DAAD).

${ }^{\ddagger}$ Department of Mathematics, Wayne State University, 656 West Kirby Avenue, Detroit, MI 48202 (boris@math.wayne.edu). This author acknowledges partial support of the U.S. National Science Foundation under grant DMS-1007132, by the European Regional Development Fund (FEDER), and by the following Portuguese agencies: Foundation for Science and Technologies (FCT), Operational Program for Competitiveness Factors (COMPETE), and Strategic Reference Framework (QREN). 
respectively, with $G: \mathbb{R}^{n} \rightarrow \mathbb{R}^{k}$ and $g: \mathbb{R}^{n} \times \mathbb{R}^{m} \rightarrow \mathbb{R}^{p}$. Furthermore, all the functions involved will be assumed to be continuously differentiable. The reader may observe from our analysis that most of the results obtained can be extended to the case of equality and other types of constraints as well as to the case of nonsmooth functions.

The quotation marks in (1.1) are used to express the uncertainty in the formalization of the bilevel optimization problem in the case of nonuniquely determined lower-level optimal solutions. In the latter case two major approaches have been suggested in the literature in order to easily handle the problem. On one hand, we have the optimistic formulation

$$
\min \left\{\varphi_{o}(x) \mid x \in X\right\} \text { with } \varphi_{o}(x):=\min _{y}\{F(x, y) \mid y \in S(x)\} .
$$

From the economics viewpoint this corresponds to a situation where the follower participates in the profit of the leader; i.e., some cooperation is possible between both players on the upper and lower levels. However, it would not always be possible for the leader to convince the follower to make choices that are favorable for him or her. Hence it is necessary for the upper-level player to bound damages resulting from undesirable selections on the lower level. It gives the pessimistic formulation of the bilevel optimization problem as follows:

$$
\min \left\{\varphi_{p}(x) \mid x \in X\right\} \text { with } \varphi_{p}(x):=\max _{y}\{F(x, y) \mid y \in S(x)\} .
$$

This problem belongs to a special class of minimax problems. Minimax problems have been intensively studied in the literature; see, e.g., [54, 55, 63] and the references therein. However, it has been well recognized that when $S(x)$ stands for varying sets of solutions to another optimization problem, the pessimistic formulation above faces many challenges. Some of them are highlighted in [8]. Recent developments on pessimistic bilevel programs can be found in $[4,7,12,30]$.

Our main concern in this paper is the original optimistic formulation $\left(\mathrm{P}_{o}\right)$ in bilevel programming that has been eventually substituted in the literature, under the name of "optimistic bilevel program," by the following optimization problem:

$$
\min _{x, y}\{F(x, y) \mid x \in X, y \in S(x)\} .
$$

The latter problem, which we label here as the conventional/auxiliary bilevel program, has been well investigated. In the last two decades, problem $(\mathrm{P})$ has attracted a lot of interest from both viewpoints of optimization theory and applications. The reader is referred to $[2,8,55]$ and the bibliographies therein for detailed discussions. For more recent results on the topic we refer the reader to $[6,10,11,13,16,27,41,62]$. In addition, a vast literature on related mathematical problems with equilibrium constraints (MPECs) is widely available; see the books [33, 38, 39, 47] with their commentaries and references. Note here that in investigating problem $(\mathrm{P})$ and related MPECs, researchers face the issue of passing to an equivalent single-level reformulation, especially when the so-called Karush-Kuhn-Tucker (KKT) reformulation is in question [10]. As will be made clear in this paper, investigating the bilevel program $\left(\mathrm{P}_{o}\right)$ of our main interest in what follows does not lead to such a difficulty.

Unfortunately, very little is known about the initial bilevel program $\left(\mathrm{P}_{o}\right)$ that is the original optimistic model in the bilevel programming problem (1.1) and is labeled as such. It has been well recognized that problems $\left(\mathrm{P}_{o}\right)$ and $(\mathrm{P})$ are equivalent for global solutions while not for local ones. We show in section 6 that a local optimal solution of problem $\left(\mathrm{P}_{o}\right)$ corresponds to a local optimal solution of $(\mathrm{P})$ without any assumption. However, it is easy to find examples where a local optimal solution of $(\mathrm{P})$ does not generate a local optimal solution of $\left(\mathrm{P}_{o}\right)$; cf. section 6 . Thus we also show in 
this section that a local optimal solution of $(\mathrm{P})$ corresponds to that of $\left(\mathrm{P}_{o}\right)$ under a rather strong condition, namely, the inner semicontinuity of a certain multifunction. This clearly means that there is a gap between the original optimistic bilevel program $\left(\mathrm{P}_{o}\right)$ and the auxiliary model $(\mathrm{P})$, most importantly because they are both nonconvex optimization problems, and thus finding local optimal solutions is the most likely achievable goal in practical solution methods. It is clear that there is no distinction between both problems in the case where optimal solutions of the lower-level problem are uniquely determined. Ruling out this possibility, a crucial question that arises is How are stationary points of $\left(\mathrm{P}_{o}\right)$ and $(\mathrm{P})$ related to each other? Among other things, we attempt to answer this question in the present paper.

To proceed in this direction, we aim to derive comprehensive first-order necessary optimality conditions, via various types of stationarity in bilevel programming, for the original problem $\left(\mathrm{P}_{o}\right)$ and compare them with known ones for $(\mathrm{P})$. According to the general approach to "abstract" problems of this type developed in [38, 39], sensitivity analysis and necessary optimality conditions for such problems are closely related to deriving appropriate subdifferential estimates for the optimal value function

$$
\varphi_{o}(x):=\min _{y}\{F(x, y) \mid y \in S(x)\} .
$$

We assume with no further mentioning that the minimum in (1.4) and similar settings below is realized. In the framework of this paper the value function (1.4) is not just defined via an abstract mapping $S$ but it is also associated with the two-level optimization problem $\left(\mathrm{P}_{o}\right)$, where $S$ is the solution map of the specifically given lowerlevel problem of parametric optimization. For this reason we call (1.4) the two-level value function.

A large literature exists for value functions (known also as marginal functions) in conventional optimization problems with inequalities and/or equalities; see, e.g., $[3,5,19,23,50]$ to name just a few. Since marginal functions are intrinsically nonsmooth, generalized derivatives of various kinds are used to study their properties. More recently, significant progress in the study and applications of various classes of marginal/value functions has been made by using generalized differential constructions introduced by the second author [35]; more details can be found in $[11,16,38,39,40,41,42,43]$. Note that in problems of nonlinear and nondifferentiable programming the key conditions needed for subdifferential estimates and sensitivity analysis of the corresponding marginal functions are the classical constraint qualification by Mangasarian and Fromovitz [34] (MFCQ) and its nonsmooth extension introduced in [37]. It happens that these qualification conditions are not applicable to the two-level value function $\varphi_{o}$ written in a marginal function form under parametric functional constraints; see section 5 . Thus adequate rules tailored for $\varphi_{o}$ have to be developed.

In order to tackle this, we consider in this paper three possible approaches to sensitivity analysis for two-level value functions of type (1.4) involving certain representations/transformations of the solution map

$$
S(x):=\underset{y}{\operatorname{argmin}}\{f(x, y) \mid y \in K(x)\}
$$

of the lower-level problem in the construction of $\varphi_{o}$. Here we label them conditionally as the complementarity/OPCC approach, the generalized equation/OPEC approach, and the lower-level value function/LLVF approach. 
In the first two approaches, function (1.4) becomes the optimal value function of an optimization problem with complementarity constraints (OPCC) and of an optimization problem with a generalized equation constraint (OPEC), respectively. To the best of our knowledge, the initial results for value functions of this type were obtained by Lucet and Ye [32] and by Hu and Ralph [29]. Paper [29] is devoted to the study of strict differentiability as well as first-order and second-order directional derivatives of the value function for the corresponding OPCC under the OPCC/MPEC linear independence constraint qualification (MPEC-LICQ). Another approach is developed in [32], which employs the limiting subdifferential constructions by Mordukhovich to conduct a local sensitivity analysis of value functions of the above types.

The developments of this paper within the OPCC approach to sensitivity analysis of value functions are much closer to those by Lucet and Ye, though we also try to bridge the gap between our work and that by Hu and Ralph; see subsection 3.3. Note that the results of [32] for OPCC value functions focus only on the case where one of the functional components involved in the crucial complementarity condition is given by the simplest linear function. Some of our results obtained in section 3 can be seen as extensions of those in [32] to the general function setting in the complementarity condition. On the other hand, our results in section 4 within the OPEC approach cover the generalized equation description of (1.5) via the normal cone to moving convex sets (of the quasi-variational inequality type), which was not considered in [32]. In this way we derive more detailed upper estimates for the limiting subdifferential of the corresponding value function and establish their clear link with those obtained via the OPCC approach; see subsection 5.1. Another important difference between our work on sensitivity analysis for value functions via the OPCC and OPEC approaches and the one by Lucet and Ye is that we do not use their growth hypothesis, which plays a significant role in the results of [32]. We replace it by the inner semicompactness assumption (weaker than a certain uniform boundedness condition closely related to the aforementioned growth hypothesis) imposed on the solution map

$$
S_{o}(x):=\left\{y \in S(x) \mid F(x, y) \leq \varphi_{o}(x)\right\}
$$

and derive tighter upper bounds for the limiting subdifferential of $\varphi_{o}$ under the inner semicontinuity of $S_{o}(1.6)$; see section 2 for more details on these notions.

In the third (LLVF) of the aforementioned approaches, originated by Outrata [45] for a special class of bilevel programs/Stackelberg games, we represent the solution map (1.5) of the lower-level problem as an inequality system containing the lower-level value function of (1.2). In this way we provide verifiable conditions in terms of the initial data to evaluate the coderivative of $S$ and establish the Lipschitz-like property of this mapping. This leads us in turn to new conditions ensuring the local Lipschitz continuity of the two-level value function $\varphi_{o}$; see subsection 5.2 for all the details.

The rest of the paper is organized as follows. Section 2 presents basic notions and results of variational analysis and generalized differentiation widely used in the subsequent parts. Section 3 is mainly concerned with sensitivity analysis of OPCC value functions. Here we derive upper estimates of the limiting subdifferential for such functions from various perspectives, depending on the type of optimality/stationary conditions of interest for the original bilevel model $\left(\mathrm{P}_{o}\right)$. It should be mentioned that the results in section 3 can stand on their own. Indeed, they also provide efficient rules to obtain estimates of the coderivative and the fulfillment of the Lipschitz-like property for mappings of special structures (inequality and equality systems with complementarity constraints) that are important for other classes of optimization-related 
problems, not just for bilevel programming. Sensitivity analysis of OPEC value functions, which is of its own interest as well, is conducted in section 4.

The first part of section 5 mainly deals with the applications of the results from sections 3 and 4 to sensitivity analysis of the two-level value function (1.4) via the OPCC and OPEC approaches. In the second part of this section (i.e., in subsection 5.2) we develop the lower-level value function approach to analyze the two-level value function $\varphi_{o}$. Here a detailed discussion is given on rules to derive subdifferential estimates and establish the local Lipschitz continuity of $\varphi_{o}$ from a perspective completely different from the previous ones.

In the final section 6 we employ the results obtained above to derive necessary optimality conditions for the original optimistic formulation $\left(\mathrm{P}_{o}\right)$ in the various forms of stationarity conditions including the new types introduced in this paper. A clear relationship between optimal solutions of $(\mathrm{P})$ and $\left(\mathrm{P}_{o}\right)$ is also provided in this section.

2. Background material. More details on the material briefly discussed in this section can be found in the books $[38,39,51,53]$ and the references therein. We start with the Painlevé-Kuratowski outer/upper limit of a set-valued mapping $\Psi: \mathbb{R}^{n} \rightrightarrows \mathbb{R}^{m}$ as $x \rightarrow \bar{x}$ defined by

$$
\operatorname{Limsup}_{x \rightarrow \bar{x}} \Psi(x):=\left\{v \in \mathbb{R}^{m} \mid \exists x_{k} \rightarrow \bar{x}, v_{k} \rightarrow v \text { with } v_{k} \in \Psi\left(x_{k}\right) \text { as } k \rightarrow \infty\right\} .
$$

Given an extended-real-valued function $\psi: \mathbb{R}^{n} \rightarrow \overline{\mathbb{R}}:=(-\infty, \infty]$, the Fréchet/regular subdifferential of $\psi$ at $\bar{x} \in \operatorname{dom} \psi:=\left\{x \in \mathbb{R}^{n} \mid \psi(x)<\infty\right\}$ is given by

$$
\widehat{\partial} \psi(\bar{x}):=\left\{v \in \mathbb{R}^{n} \mid \liminf _{x \rightarrow \bar{x}} \frac{\psi(x)-\psi(\bar{x})-\langle v, x-\bar{x}\rangle}{\|x-\bar{x}\|} \geq 0\right\},
$$

while our basic construction in this paper known as the Mordukhovich/basic or limiting subdifferential of $\psi$ at $\bar{x} \in \operatorname{dom} \psi$ is defined via the outer limit (2.1) by

$$
\partial \psi(\bar{x}):=\operatorname{Limsup}_{x \rightarrow \bar{x}} \widehat{\partial} \psi(x) .
$$

If $\psi$ is convex, then the subdifferential $\partial \psi(\bar{x})$ reduces to the classical subdifferential of convex analysis. If $\psi$ is locally Lipschitzian around $\bar{x}$, then the set $\partial \psi(\bar{x})$ is nonempty and compact. Moreover, its convex hull agrees with the subdifferential/generalized gradient by Clarke. If $\psi$ is strictly differentiable at $\bar{x}$, i.e.,

$$
\lim _{v \rightarrow \bar{x}, x \rightarrow \bar{x}} \frac{\psi(v)-\psi(x)-\langle\nabla \psi(\bar{x}), v-x\rangle}{\|v-x\|}=0,
$$

then $\partial \psi(\bar{x})=\{\nabla \psi(\bar{x})\}$. It should be mentioned that every function continuously differentiable around some point is strictly differentiable at this point and that every function locally Lipschitzian around $\bar{x}$ is strictly differentiable at $\bar{x}$, provided that its subdifferential (2.2) is a singleton.

Given a nonempty set $\Omega \subset \mathbb{R}^{n}$, our basic normal cone to it at $\bar{x} \in \Omega$ corresponding to the subdifferential construction (2.2) is defined by

$$
N_{\Omega}(\bar{x}):=\operatorname{Limsup}_{x \rightarrow \bar{x}(x \in \Omega)} \widehat{N}_{\Omega}(x)
$$

via the outer limit (2.1) of the regular counterpart

$$
\widehat{N}_{\Omega}(x):=\left\{v \in \mathbb{R}^{n} \mid \limsup _{u \rightarrow x(u \in \Omega)} \frac{\langle v, u-x\rangle}{\|u-x\|} \leq 0\right\}
$$


at points $x \in \Omega$ near $\bar{x}$. Note that for sets $\Omega \subset \mathbb{R}^{n}$ locally closed around $\bar{x}$ the given definition (2.4) reduces to the original one,

$$
N_{\Omega}(\bar{x})=\operatorname{Limsup}_{x \rightarrow \bar{x}}\left[\operatorname{cone}\left(x-\Pi_{\Omega}(x)\right)\right],
$$

introduced in [35], where "cone" stands for the conic hull of a set, and where $\Pi$ denotes the Euclidean projection on the set in question. Using the normal cone (2.4), we can equivalently describe the basic subdifferential (2.2) by

$$
\partial \psi(\bar{x})=\left\{v \in \mathbb{R}^{n} \mid(v,-1) \in N_{\mathrm{epi} \psi}(\bar{x}, \psi(\bar{x}))\right\}
$$

for lower semicontinuous (l.s.c.) functions with the epigraph epi $\psi$ and define the singular subdifferential of $\psi$ at $\bar{x} \in \operatorname{dom} \psi$ by

$$
\partial^{\infty} \psi(\bar{x}):=\left\{v \in \mathbb{R}^{n} \mid(v, 0) \in N_{\mathrm{epi} \psi}(\bar{x}, \psi(\bar{x}))\right\} .
$$

It is worth mentioning that for functions $\psi$ l.s.c. around $\bar{x}$ we have $\partial^{\infty} \psi(\bar{x})=\{0\}$ if and only if $\psi$ is locally Lipschitzian around this point.

Given further a set-valued mapping $\Psi: \mathbb{R}^{n} \rightrightarrows \mathbb{R}^{m}$ with the graph

$$
\operatorname{gph} \Psi:=\left\{(x, y) \in \mathbb{R}^{n} \times \mathbb{R}^{m} \mid y \in \Psi(x)\right\},
$$

recall the notion of coderivative for $\Psi$ at $(\bar{x}, \bar{y}) \in$ gph $\Psi$ defined in [36] by

$$
D^{*} \Psi(\bar{x}, \bar{y})(v):=\left\{u \in \mathbb{R}^{n} \mid(u,-v) \in N_{\operatorname{gph} \Psi}(\bar{x}, \bar{y})\right\} \text { for } v \in \mathbb{R}^{m}
$$

via the normal cone (2.4) to the graph of $\Psi$. If $\Psi$ is single-valued and locally Lipschitzian around $\bar{x}$, its coderivative can be represented analytically as

$$
D^{*} \Psi(\bar{x})(v)=\partial\langle v, \Psi\rangle(\bar{x}) \text { for } v \in \mathbb{R}^{m}
$$

via the basic subdifferential (2.2) of the Lagrange scalarization $\langle v, \Psi\rangle(x):=\langle v, \Psi(x)\rangle$, where the component $\bar{y}(=\Psi(\bar{x}))$ is omitted in the coderivative notation for singlevalued mappings. This implies the coderivative representation

$$
D^{*} \Psi(\bar{x})(v)=\left\{\nabla \Psi(\bar{x})^{\top} v\right\} \text { for } v \in \mathbb{R}^{m}
$$

when $\Psi$ is strictly differentiable at $\bar{x}$ as in (2.3) with $\nabla \Psi(\bar{x})$ standing for its Jacobian matrix at $\bar{x}$ and with the symbol "T" standing for transposition.

Some continuity properties of set-valued mappings are of a particular interest in this paper. We say that $\Psi: \mathbb{R}^{n} \rightrightarrows \mathbb{R}^{m}$ is inner semicompact at $\bar{x}$ with $\Psi(\bar{x}) \neq \emptyset$ if for every sequence $x_{k} \rightarrow \bar{x}$ with $\Psi\left(x_{k}\right) \neq \emptyset$ there is a sequence of $y_{k} \in \Psi\left(x_{k}\right)$ that contains a convergent subsequence. It follows that the inner semicompactness holds in finite dimensions whenever $\Psi$ is uniformly bounded around $\bar{x}$; i.e., there exists a neighborhood $U$ of $\bar{x}$ and a bounded set $C \subset \mathbb{R}^{m}$ such that

$$
\Psi(x) \subset C \forall x \in U .
$$

The mapping $\Psi$ is inner semicontinuous at $(\bar{x}, \bar{y}) \in$ gph $\Psi$ if for every sequence $x_{k} \rightarrow \bar{x}$ there is a sequence of $y_{k} \in \Psi\left(x_{k}\right)$ that converges to $\bar{y}$ as $k \rightarrow \infty$. This property reduces to the usual continuity for single-valued mappings, while in the set-valued case it is 
implied by the Lipschitz-like/Aubin property of $\Psi$ around $(\bar{x}, \bar{y}) \in \operatorname{gph} \Psi$, which means that there are neighborhoods $U$ of $\bar{x}, V$ of $\bar{y}$, and a constant $\ell>0$ such that

$$
d(y ; \Psi(x)) \leq \ell\|x-u\| \forall x, u \in U \text { and } \forall y \in \Psi(u) \cap V,
$$

where $d$ stands for the usual distance function. When $V=\mathbb{R}^{m}$ in (2.8), this property reads as to the classical local Lipschitz continuity of $\Psi$ around $\bar{x}$. A complete characterization of the Lipschitz-like property (2.8), and hence a sufficient condition for the inner semicontinuity of $\Phi$ at $(\bar{x}, \bar{y})$, is given for closed-graph mappings by the following coderivative/Mordukhovich criterion (see [39, Theorem 5.7] and [51, Theorem 9.40]):

$$
D^{*} \Psi(\bar{x}, \bar{y})(0)=\{0\} .
$$

Furthermore, the infimum of all $\ell>0$ for which (2.8) holds is equal to the coderivative norm $\left\|D^{*} \Psi(\bar{x}, \bar{y})\right\|$ as a positively homogeneous mapping $D^{*} \Psi(\bar{x}, \bar{y}): \mathbb{R}^{m} \rightrightarrows \mathbb{R}^{n}$.

If we fix $x=\bar{x}$ in (2.8), the resulting weaker property is known as calmness of $\Psi$ at $(\bar{x}, \bar{y})[51]$; for $V=\mathbb{R}^{m}$ it reduces to the upper Lipschitz property of Robinson [49].

In order to analyze our two-level optimal value function $\varphi_{o}$ in (1.4), we first consider a general "abstract" framework of the marginal functions

$$
\mu(x):=\min _{y}\{\psi(x, y) \mid y \in \Psi(x)\}
$$

with $\psi: \mathbb{R}^{n} \times \mathbb{R}^{m} \rightarrow \overline{\mathbb{R}}$ and $\Psi: \mathbb{R}^{n} \rightrightarrows \mathbb{R}^{m}$. Consider the argminimum mapping

$$
\Psi_{o}(x):=\operatorname{argmin}\{\psi(x, y) \mid y \in \Psi(x)\}=\{y \in \Psi(x) \mid \psi(x, y) \leq \mu(x)\}
$$

and summarize in the next theorem some known results on general marginal functions needed in the paper; see [38, Corollary 1.109] and [39, Theorem 5.2].

THEOREM 2.1 (properties of general marginal functions). Let the marginal function $\mu$ be given in (2.10), where the graph of $\Psi$ is locally closed around $(\bar{x}, \bar{y}) \in \operatorname{gph} \Psi$, and where $\psi$ is strictly differentiable at this point. The following assertions hold:

(i) Let $\Psi_{o}$ be inner semicontinuous at $(\bar{x}, \bar{y})$. Then $\mu$ is lower semicontinuous at $\bar{x}$ and we have the following upper bound for its basic subdifferential:

$$
\partial \mu(\bar{x}) \subset \nabla_{x} \psi(\bar{x}, \bar{y})+D^{*} \Psi(\bar{x}, \bar{y})\left(\nabla_{y} \psi(\bar{x}, \bar{y})\right) .
$$

If in addition $\Psi$ is Lipschitz-like around $(\bar{x}, \bar{y})$, then we also have the Lipschitz continuity of $\mu$ around $\bar{x}$.

(ii) Let $\Psi_{o}$ be inner semicompact at $\bar{x}$. Then $\mu$ is lower semicontinuous at $\bar{x}$ and

$$
\partial \mu(\bar{x}) \subset \bigcup_{\bar{y} \in \Psi_{o}(\bar{x})}\left\{\nabla_{x} \psi(\bar{x}, \bar{y})+D^{*} \Psi(\bar{x}, \bar{y})\left(\nabla_{y} \psi(\bar{x}, \bar{y})\right)\right\} .
$$

If in addition $\Psi$ is Lipschitz-like around $(\bar{x}, \bar{y})$ for all vectors $\bar{y} \in \Psi_{o}(\bar{x})$, then $\mu$ is Lipschitz continuous around $\bar{x}$.

Depending on specific structures of the set-valued mapping $\Psi$, our aim in sections $3-5$ is to give detailed upper bounds for $D^{*} \Psi(\bar{x}, \bar{y})$ in terms of the problem data and to provide verifiable rules for the Lipschitz-like property of $\Psi$, which imply explicit upper bounds for $\partial \mu(\bar{x})$ and the local Lipschitz continuity of $\mu$. More discussions on the inner semicontinuity of argminimum mappings can be found in [11, Remark 3.2].

To conclude this section, we present a constraint qualification and necessary optimality conditions for a general optimization problem with geometric constraints in terms of limiting normals and subgradients; see, e.g., [39, Proposition 5.3].

Copyright (c) by SIAM. Unauthorized reproduction of this article is prohibited. 
TheOREM 2.2 (optimality conditions under geometric constraints). Let $\bar{x}$ be a local optimal solution to the problem

$$
\text { minimize } \psi(x) \text { subject to } x \in \Omega \text {, }
$$

where $\psi: \mathbb{R}^{n} \rightarrow \overline{\mathbb{R}}$ is l.s.c. around $\bar{x} \in \Omega \cap \operatorname{dom} \psi$, and where $\Omega \subset \mathbb{R}^{n}$ is locally closed around this point. Then we have

$$
0 \in \partial \psi(\bar{x})+N_{\Omega}(\bar{x})
$$

provided the validity of the qualification condition

$$
\partial^{\infty} \psi(\bar{x}) \cap\left(-N_{\Omega}(\bar{x})\right)=\{0\},
$$

which is the case, in particular, when $\psi$ is locally Lipschitzian around $\bar{x}$.

3. Sensitivity analysis of OPCC value functions. In this section we consider the value function of a parametric optimization problem belonging to the class of optimization programs with complementarity constraints (OPCCs):

$$
\begin{aligned}
\mu^{c}(x):=\min _{y}\left\{F(x, y) \mid y \in S^{c}(x)\right. & :=\{y \mid g(x, y) \\
G(x, y) & \left.\left.\geq 0, H(x, y) \geq 0, G(x, y)^{\top} H(x, y)=0\right\}\right\},
\end{aligned}
$$

where $F: \mathbb{R}^{n} \times \mathbb{R}^{m} \rightarrow \mathbb{R}, g: \mathbb{R}^{n} \times \mathbb{R}^{m} \rightarrow \mathbb{R}^{a}, h: \mathbb{R}^{n} \times \mathbb{R}^{m} \rightarrow \mathbb{R}^{b}$, and $G, H: \mathbb{R}^{n} \times \mathbb{R}^{m} \rightarrow$ $\mathbb{R}^{d}$ are all continuously differentiable functions. The main goal of this section is deriving efficient subdifferential estimates for the optimal value function (3.1), as well as verifiable conditions for its local Lipschitz continuity and for the Lipschitz-like property of the feasible solution map $S^{c}$ entirely in terms of the initial data. Adopting the terminology originated by Scheel and Scholtes [52], the sensitivity analysis results established below and the associated constraint qualifications are expressed via the sets of M-, C-, and S-type multipliers used in the corresponding M(ordukhovich), $C$ (larke), and S(trong) stationarity conditions for OPCCs; cf. section 6.

Fix a pair $(\bar{x}, \bar{y}) \in \operatorname{gph} S^{c}$ and associate with it the following partition of the indices for the functions involved in the complementarity system in (3.1):

$$
\begin{array}{ll}
\eta=\eta(\bar{x}, \bar{y}):=\{i=1, \ldots, d \mid & \left.G_{i}(\bar{x}, \bar{y})=0, H_{i}(\bar{x}, \bar{y})>0\right\} \\
\theta=\theta(\bar{x}, \bar{y}):=\{i=1, \ldots, d \mid & \left.G_{i}(\bar{x}, \bar{y})=0, H_{i}(\bar{x}, \bar{y})=0\right\} \\
\nu=\nu(\bar{x}, \bar{y}):=\{i=1, \ldots, d \mid & \left.G_{i}(\bar{x}, \bar{y})>0, H_{i}(\bar{x}, \bar{y})=0\right\}
\end{array}
$$

where the set $\theta$ in (3.2) is known as the biactive or degenerate index set. As shown below, the difference between the various types of multiplier sets depends on the structure of the components corresponding to the biactive set $\theta$. Now consider a vector $v \in \mathbb{R}^{n+m}$ and define the set of M-type multipliers associated with (3.1) by

$$
\begin{gathered}
\Lambda^{c m}(\bar{x}, \bar{y}, v):=\left\{(\alpha, \beta, \gamma, \zeta) \mid \alpha \geq 0, \alpha^{\top} g(\bar{x}, \bar{y})=0,\right. \\
\quad \gamma_{\nu}=0, \zeta_{\eta}=0, \\
\forall i \in \theta,\left(\gamma_{i}<0 \wedge \zeta_{i}<0\right) \vee \gamma_{i} \zeta_{i}=0, \\
\left.v+\nabla g(\bar{x}, \bar{y})^{\top} \alpha+\nabla h(\bar{x}, \bar{y})^{\top} \beta+\nabla G(\bar{x}, \bar{y})^{\top} \gamma+\nabla H(\bar{x}, \bar{y})^{\top} \zeta=0\right\} .
\end{gathered}
$$

Similarly we define the set $\Lambda_{y}^{c m}(\bar{x}, \bar{y}, v)$, with $v \in \mathbb{R}^{m}$, obtained by replacing the gradients of $g, h, G$, and $H$ in equation $v+\nabla g(\bar{x}, \bar{y})^{\top} \alpha+\nabla h(\bar{x}, \bar{y})^{\top} \beta+\nabla G(\bar{x}, \bar{y})^{\top} \gamma+$ $\nabla H(\bar{x}, \bar{y})^{\top} \zeta=0$ by their partial derivatives with respect to $y$. In the case where $v:=\nabla_{y} F(\bar{x}, \bar{y})$ we denote $\Lambda_{y}^{c m}(\bar{x}, \bar{y}):=\Lambda_{y}^{c m}\left(\bar{x}, \bar{y}, \nabla_{y} F(\bar{x}, \bar{y})\right)$. 
The corresponding sets of $C$-type multipliers denoted by $\Lambda^{c c}(\bar{x}, \bar{y}, v), \Lambda_{y}^{c c}(\bar{x}, \bar{y}, v)$, and $\Lambda_{y}^{c c}(\bar{x}, \bar{y})$ are defined similarly to $\Lambda^{c m}(\bar{x}, \bar{y}, v), \Lambda_{y}^{c m}(\bar{x}, \bar{y}, v)$, and $\Lambda_{y}^{c m}(\bar{x}, \bar{y})$, respectively, with the replacement of condition $\left(\gamma_{i}<0 \wedge \zeta_{i}<0\right) \vee \gamma_{i} \zeta_{i}=0$ on $\theta$ by $\gamma_{i} \zeta_{i} \geq 0$ on the same set. For the case of $S$-type multipliers we need to define only $\Lambda_{y}^{c s}(\bar{x}, \bar{y})$, which is an analogue of $\Lambda_{y}^{c m}(\bar{x}, \bar{y})$ with the replacement of the aforementioned condition therein by $\gamma_{i} \leq 0 \wedge \zeta_{i} \leq 0$ for all $i \in \theta$. The following links between the sets $\Lambda_{y}^{c s}(\bar{x}, \bar{y}), \Lambda_{y}^{c m}(\bar{x}, \bar{y})$, and $\Lambda_{y}^{c c}(\bar{x}, \bar{y})$ are obvious: $\Lambda_{y}^{c s}(\bar{x}, \bar{y}) \subset \Lambda_{y}^{c m}(\bar{x}, \bar{y}) \subset \Lambda_{y}^{c c}(\bar{x}, \bar{y})$.

To further simplify the presentation of this section, we introduce the following Lagrange-type and singular Lagrange-type functions, respectively, associated with the parametric problem in (3.1):

$$
\begin{aligned}
L(x, y, \alpha, \beta, \gamma, \zeta) & :=F(x, y)+g(x, y)^{\top} \alpha+h(x, y)^{\top} \beta+G(x, y)^{\top} \gamma+H(x, y)^{\top} \zeta \\
L_{o}(x, y, \alpha, \beta, \gamma, \zeta) & :=g(x, y)^{\top} \alpha+h(x, y)^{\top} \beta+G(x, y)^{\top} \gamma+H(x, y)^{\top} \zeta .
\end{aligned}
$$

In what follows, the derivative of $L_{o}$ with respect to $(x, y)$ is often needed and is denoted by

$$
\nabla L_{o}(x, y, \alpha, \beta, \gamma, \zeta):=\nabla g(x, y)^{\top} \alpha+\nabla h(x, y)^{\top} \beta+\nabla G(x, y)^{\top} \gamma+\nabla H(x, y)^{\top} \zeta .
$$

The following solution map for the OPCC related to (3.1) given by

$$
S_{o}^{c}(x):=\left\{y \in S^{c}(x) \mid F(x, y) \leq \mu^{c}(x)\right\}
$$

plays a significant role in our subsequent sensitivity analysis in this section.

3.1. Sensitivity analysis via M-type multipliers. To proceed in this subsection, we define the $M$-qualification conditions at $(\bar{x}, \bar{y})$ :

$$
\begin{array}{ll}
\left(A_{1}^{1}\right) & (\alpha, \beta, \gamma, \zeta) \in \Lambda^{c m}(\bar{x}, \bar{y}, 0) \Longrightarrow \alpha=0, \beta=0, \gamma=0, \zeta=0 \\
\left(A_{2}^{1}\right) & (\alpha, \beta, \gamma, \zeta) \in \Lambda_{y}^{c m}(\bar{x}, \bar{y}, 0) \Longrightarrow \nabla_{x} L_{o}(\bar{x}, \bar{y}, \alpha, \beta, \gamma, \zeta)=0 \\
\left(A_{3}^{1}\right) & (\alpha, \beta, \gamma, \zeta) \in \Lambda_{y}^{c m}(\bar{x}, \bar{y}, 0) \Longrightarrow \alpha=0, \beta=0, \gamma=0, \zeta=0
\end{array}
$$

We observe the obvious links between them: $\left(A_{2}^{1}\right) \Longleftarrow\left(A_{3}^{1}\right) \Longrightarrow\left(A_{1}^{1}\right)$. The next theorem provides a constructive upper estimate of the coderivative (2.6) of the OPCC feasible solution map $S^{c}$ and gives a verifiable condition for its robust Lipschitzian stability, i.e., the validity of the Lipschitz-like property.

THEOREM 3.1 (coderivative estimate and Lipschitz-like property of OPCC feasible solutions via M-multipliers). Let $(\bar{x}, \bar{y}) \in$ gph $S^{c}$, and let $\left(A_{1}^{1}\right)$ hold at $(\bar{x}, \bar{y})$. Then for all $v \in \mathbb{R}^{m}$ we have

$$
D^{*} S^{c}(\bar{x}, \bar{y})(v) \subset\left\{\nabla_{x} L_{o}(\bar{x}, \bar{y}, \alpha, \beta, \gamma, \zeta) \mid(\alpha, \beta, \gamma, \zeta) \in \Lambda_{y}^{c m}(\bar{x}, \bar{y}, v)\right\} .
$$

If in addition $\left(A_{2}^{1}\right)$ is satisfied at $(\bar{x}, \bar{y})$, then $S^{c}$ is Lipschitz-like around this point.

Proof. We start by recalling that the complementarity system contained in (3.1) is equivalent to the inclusion $\left(G_{i}(x, y), H_{i}(x, y)\right) \in\left\{(u, v) \in \mathbb{R}^{2} \mid u \geq 0, v \geq\right.$ $\left.0, u^{\top} v=0\right\}:=\Lambda_{i}$ for $i=1, \ldots, d$, and the graph of $S^{c}$ can be rewritten in the form $\operatorname{gph} S^{c}=\{(x, y) \mid \psi(x, y) \in \Lambda\}$ via the vector-valued function $\psi$ and the polyhedral set $\Lambda$ defined by

$$
\psi(x, y):=\left[g(x, y), h(x, y),\left(G_{i}(x, y), H_{i}(x, y)\right)_{i=1}^{d}\right] \text { and } \Lambda:=\mathbb{R}_{-}^{a} \times\left\{0_{b}\right\} \times \prod_{i=1}^{d} \Lambda_{i} .
$$

Copyright (c) by SIAM. Unauthorized reproduction of this article is prohibited. 
It follows from the calculus rules in [38, Theorem 3.8] and [51, Theorem 6.14] that

$$
N_{\mathrm{gph} S^{c}}(\bar{x}, \bar{y}) \subset \nabla \psi(\bar{x}, \bar{y})^{\top} N_{\Lambda}(\psi(\bar{x}, \bar{y}))
$$

provided the validity of the qualification condition

$$
\left.\begin{array}{l}
\nabla \psi(\bar{x}, \bar{y})^{\top}\left(\alpha, \beta,\left(\gamma_{i}, \zeta_{i}\right)_{i=1}^{d}\right)=0 \\
\left(\alpha, \beta,\left(\gamma_{i}, \zeta_{i}\right)_{i=1}^{d}\right) \in N_{\Lambda}(\psi(\bar{x}, \bar{y}))
\end{array}\right\} \Longrightarrow \alpha=0, \beta=0, \gamma=0, \zeta=0 .
$$

It is easy to check that we have $\nabla \psi(\bar{x}, \bar{y})^{\top}\left(\alpha, \beta,\left(\gamma_{i}, \zeta_{i}\right)_{i=1}^{d}\right)=\nabla L_{o}(\bar{x}, \bar{y}, \alpha, \beta, \gamma, \zeta)$ for any quadruple $(\alpha, \beta, \gamma, \zeta)$ and that, by the product formula for limiting normals,

$$
N_{\Lambda}(\psi(\bar{x}, \bar{y}))=N_{\mathbb{R}_{-}^{a}}(g(\bar{x}, \bar{y})) \times N_{\left\{0_{b}\right\}}(h(\bar{x}, \bar{y})) \times \prod_{i=1}^{d} N_{\Lambda_{i}}\left(G_{i}(\bar{x}, \bar{y}), H_{i}(\bar{x}, \bar{y})\right) .
$$

Using the expression of the normal cone to the sets $\Lambda_{i}, i=1, \ldots, d$, from [21] (cf. also $[46,57]$ for particular cases), we get

$$
\begin{aligned}
N_{\Lambda_{i}}\left(G_{i}(\bar{x}, \bar{y}), H_{i}(\bar{x}, \bar{y})\right)= & \left\{\left(\gamma_{i}, \zeta_{i}\right) \mid \gamma_{i}=0 \text { if } i \in \nu, \zeta_{i}=0 \text { if } i \in \eta\right. \\
& \left.\left(\gamma_{i}<0, \zeta_{i}<0\right) \vee\left(\gamma_{i} \zeta_{i}=0\right) \text { if } i \in \theta\right\},
\end{aligned}
$$

which implies that the qualification condition (3.8) reduces to $\left(A_{1}^{1}\right)$ in this case and that inclusion (3.6) results from (3.7) and the coderivative definition (2.6). Finally, the Lipschitz-like property of $S^{c}$ around $(\bar{x}, \bar{y})$ under the additional M-qualification condition $\left(A_{2}^{1}\right)$ follows from (3.6) due to the coderivative criterion (2.9).

Now we can readily get efficient estimates of the limiting subdifferential of the value function (3.1) and verifiable conditions for its local Lipschitz continuity.

ThEOREM 3.2 (M-type sensitivity analysis for OPCC value functions). The following assertions hold for the value function $\mu^{c}$ in (3.1):

(i) Let the argminimum mapping $S_{o}^{c}$ from (3.4) be inner semicontinuous at $(\bar{x}, \bar{y})$, and let $\left(A_{1}^{1}\right)$ hold at $(\bar{x}, \bar{y})$. Then we have the subdifferential upper estimate

$$
\partial \mu^{c}(\bar{x}) \subset\left\{\nabla_{x} L(\bar{x}, \bar{y}, \alpha, \beta, \gamma, \zeta) \mid(\alpha, \beta, \gamma, \zeta) \in \Lambda_{y}^{c m}(\bar{x}, \bar{y})\right\} .
$$

If in addition $\left(A_{2}^{1}\right)$ is satisfied at $(\bar{x}, \bar{y})$, then $\mu^{c}$ is Lipschitz continuous around $\bar{x}$.

(ii) Assume that $S_{o}^{c}$ is inner semicompact at $\bar{x}$ and that $\left(A_{1}^{1}\right)$ holds at $(\bar{x}, \bar{y})$ for all $\bar{y} \in S_{o}^{c}(x)$. Then we have the subdifferential upper estimate

$$
\partial \mu^{c}(\bar{x}) \subset\left\{\nabla_{x} L(\bar{x}, \bar{y}, \alpha, \beta, \gamma, \zeta) \mid \bar{y} \in S_{o}^{c}(\bar{x}),(\alpha, \beta, \gamma, \zeta) \in \Lambda_{y}^{c m}(\bar{x}, \bar{y})\right\} .
$$

If in addition $\left(A_{2}^{1}\right)$ is satisfied at $(\bar{x}, \bar{y})$ for all $\bar{y} \in S_{o}^{c}(\bar{x})$, then the value function $\mu^{c}$ is Lipschitz continuous around $\bar{x}$.

Proof. The proof follows from the results of Theorems 3.1 and 2.1.

Note that a subdifferential upper estimate similar to (ii) in Theorem 3.2 was obtained in [32] in the case of $G(x, y):=y$ under a certain growth hypothesis, which is similar to our uniform boundedness condition (2.7) on the mapping $S_{o}^{c}$ from (3.4).

Remark 3.3 (qualification conditions). There are various sufficient conditions for the validity of $\left(A_{1}^{1}\right)$; see, e.g., [14] for related developments. Furthermore, $\left(A_{1}^{1}\right)$ can be replaced by the weaker calmness assumption on the mapping

$$
\Psi(v):=\{(x, y) \mid \psi(x, y)+v \in \Lambda\},
$$

Copyright $@$ by SIAM. Unauthorized reproduction of this article is prohibited. 
where $\psi$ and $\Lambda$ are defined in the proof of Theorem 3.1. Indeed, it is shown in [24, Theorem 4.1] that the calmness of (3.9) is sufficient for inclusion (3.7), which thus ensures the conclusions of Theorems 3.1 and 3.2 by the proofs above. Note that the latter calmness assumption automatically holds when the mappings $g, h, G$, and $H$ are linear. Observe finally that due to the relationships between the constraint qualifications in (3.5) both assumptions $\left(A_{1}^{1}\right)$ and $\left(A_{2}^{1}\right)$ in the above results can be replaced by the fulfillment of the single condition $\left(A_{3}^{1}\right)$.

Remark 3.4 (relationships to previous work on the topic). Recall here that the technique employed in Theorem 3.1 that transforms the complementarity system in (3.1) into an inclusion is rather common in the field of OPCCs for studying some issues different from those considered here; see, e.g., [46, 57, 60] for related developments. Note, however, that some differences occur in constructing the set $\Lambda$ corresponding, in the proof of Theorem 3.1, to $\left\{(u, v) \in \mathbb{R}^{d} \mid u \geq 0, v \geq 0, u^{\top} v=0\right\}$, while in the aforementioned papers $\Lambda=\operatorname{gph} N_{R_{+}^{d}}$ is often chosen. Note also in [28] a transformation in this vein is employed to derive an exact penalty result and then optimality conditions for the so-called mathematical programs with vanishing constraints [1]. Having in mind this transformation, the methods developed here (cf., in particular, the proofs of Theorems 3.1 and 3.2) can readily be applied to conduct a local sensitivity analysis for the latter class of programs.

3.2. Sensitivity analysis via C-type multipliers. Similarly to subsection 3.1 we introduce the following $C$-qualification conditions at $(\bar{x}, \bar{y})$ :

$$
\begin{array}{ll}
\left(A_{1}^{2}\right) & (\alpha, \beta, \gamma, \zeta) \in \Lambda^{c c}(\bar{x}, \bar{y}, 0) \Longrightarrow \alpha=0, \beta=0, \gamma=0, \zeta=0 \\
\left(A_{2}^{2}\right) & \left.(\alpha, \beta, \gamma, \zeta) \in \Lambda_{y}^{c c}(\bar{x}, \bar{y}, 0) \Longrightarrow \nabla_{x} L_{o} \bar{x}, \bar{y}, \alpha, \beta, \gamma, \zeta\right)=0 \\
\left(A_{3}^{2}\right) & (\alpha, \beta, \gamma, \zeta) \in \Lambda_{y}^{c c}(\bar{x}, \bar{y}, 0) \Longrightarrow \alpha=0, \beta=0, \gamma=0, \zeta=0
\end{array}
$$

with a similar relationships between them: $\left(A_{2}^{2}\right) \Longleftarrow\left(A_{3}^{2}\right) \Longrightarrow\left(A_{1}^{2}\right)$. To proceed, we use the well-known nonsmooth transformation of the feasible set to the OPCC introduced by Scheel and Scholtes [52]:

$$
\begin{aligned}
S^{c}(x):=\left\{y \in \mathbb{R}^{m} \mid\right. & g(x, y) \leq 0, h(x, y)=0, \\
& \left.\min \left\{G_{i}(x, y), H_{i}(x, y)\right\}=0, i=1, \ldots, d\right\} .
\end{aligned}
$$

Employing (3.11), a C-counterpart of Theorem 3.2 can be derived with a different proof and a larger estimate for the coderivative of $S^{c}$ under $\left(A_{1}^{2}\right)$ and $\left(A_{2}^{2}\right)$.

THEOREM 3.5 (coderivative estimate and Lipschitz-like property of OPCC feasible solutions via C-multipliers). Let $\left(A_{1}^{2}\right)$ hold at $(\bar{x}, \bar{y}) \in g p h S^{c}$. Then

$$
D^{*} S^{c}(\bar{x}, \bar{y})(v) \subset\left\{\nabla_{x} L_{o}(\bar{x}, \bar{y}, \alpha, \beta, \gamma, \zeta) \mid(\alpha, \beta, \gamma, \zeta) \in \Lambda_{y}^{c c}(\bar{x}, \bar{y}, v)\right\}, \quad v \in \mathbb{R}^{m} .
$$

If in addition $\left(A_{2}^{2}\right)$ is satisfied at $(\bar{x}, \bar{y})$, then $S^{c}$ is Lipschitz-like around this point.

Proof. From the expression of $S^{c}$ in (3.11) we get gph $S^{c}=\{(x, y) \mid \psi(x, y) \in \Lambda\}$, where $\psi$ and $\Lambda$ are defined by

$$
\psi(x, y):=[g(x, y), h(x, y), V(x, y)] \text { and } \Lambda:=\mathbb{R}_{-}^{a} \times\left\{0_{b}\right\} \times\left\{0_{d}\right\}
$$

with $V_{i}(x, y):=\min \left\{G_{i}(x, y), H_{i}(x, y)\right\}=0$ for $i=1, \ldots, d$. Since $\psi$ is locally Lipschitzian around $(\bar{x}, \bar{y})$, it follows from [38, Theorem 3.8] that

$$
N_{\operatorname{gph} S^{c}}(\bar{x}, \bar{y}) \subset\left\{\partial\langle u, \psi\rangle(\bar{x}, \bar{y}) \mid u \in N_{\Lambda}(\psi(\bar{x}, \bar{y}))\right\},
$$


provided that the qualification condition

$$
\left[0 \in \partial\langle u, \psi\rangle(\bar{x}, \bar{y}), \quad u \in N_{\Lambda}(\psi(\bar{x}, \bar{y}))\right] \Longrightarrow u=0
$$

is satisfied. Furthermore, we have the normal cone representation

$$
N_{\Lambda}(\psi(\bar{x}, \bar{y}))=N_{\mathbb{R}_{-}^{a}}(g(\bar{x}, \bar{y})) \times N_{\left\{0_{b}\right\}}(h(\bar{x}, \bar{y})) \times N_{\left\{0_{d}\right\}}(V(\bar{x}, \bar{y}))
$$

and calculate the subdifferential of the scalarization in (3.13) by

$$
\partial\langle(\alpha, \beta, \chi), \psi\rangle(\bar{x}, \bar{y})=\nabla g(\bar{x}, \bar{y})^{\top} \alpha+\nabla h(\bar{x}, \bar{y})^{\top} \beta+\partial\langle\chi, V\rangle(\bar{x}, \bar{y})
$$

for $(\alpha, \beta, \chi) \in N_{\Lambda}(\psi(\bar{x}, \bar{y}))$. Since the function $V$ is nondifferentiable and $\chi$ may contain negative components by (3.15), we apply the convex hull "co" to our basic subdifferential (2.2) in (3.16) in order to instate the plus/minus symmetry

$$
\partial\langle\chi, V\rangle(\bar{x}, \bar{y}) \subset \operatorname{co} \partial\langle\chi, V\rangle(\bar{x}, \bar{y}) \subset \sum_{i=1}^{d} \chi_{i} \bar{\partial} V_{i}(\bar{x}, \bar{y})
$$

via Clarke's generalized gradient $\bar{\partial} V_{i}$. Considering the partition of the index set $\{1, \ldots, d\}$ in $(3.2)$, we arrive by [5] at the following calculations:

$$
\bar{\partial} V_{i}(\bar{x}, \bar{y})= \begin{cases}\nabla G_{i}(\bar{x}, \bar{y}) & \text { if } i \in \eta, \\ \nabla H_{i}(\bar{x}, \bar{y}) & \text { if } i \in \nu, \\ \operatorname{co}\left\{\nabla G_{i}(\bar{x}, \bar{y}), \nabla H_{i}(\bar{x}, \bar{y})\right\} & \text { if } i \in \theta .\end{cases}
$$

Invoking the classical Carathéodory theorem gives us

$$
\operatorname{co}\left\{\nabla G_{i}(\bar{x}, \bar{y}), \nabla H_{i}(\bar{x}, \bar{y})\right\}=\left\{t_{i} \nabla G_{i}(\bar{x}, \bar{y})+\left(1-t_{i}\right) \nabla H_{i}(\bar{x}, \bar{y}) \mid t_{i} \in[0,1]\right\},
$$

and hence we obtain from (3.16) the inclusions

$$
\begin{aligned}
& \partial\langle(\alpha, \beta, \chi), \psi\rangle(\bar{x}, \bar{y}) \subset\left\{\nabla L_{o}(\bar{x}, \bar{y}, \alpha, \beta, \gamma, \zeta) \mid \gamma_{\eta}=0, \zeta_{\nu}=0,\right. \\
& \left.\forall i \in \theta, \exists t_{i} \in[0,1], r_{i} \in \mathbb{R} \text { s.t. } \gamma_{i}=r_{i} t_{i}, \zeta_{i}=r_{i}\left(1-t_{i}\right)\right\} \\
& \subset\left\{\nabla L_{o}(\bar{x}, \bar{y}, \alpha, \beta, \gamma, \zeta) \mid \gamma_{\eta}=0, \zeta_{\nu}=0,\right. \\
& \left.\forall i \in \theta, \gamma_{i} \zeta_{i} \geq 0\right\} \text {. }
\end{aligned}
$$

Since the qualification condition (3.14) is equivalent to

$$
\left\{(\alpha, \beta, \chi) \mid 0 \in \partial\langle(\alpha, \beta, \chi), \psi\rangle(\bar{x}, \bar{y}),(\alpha, \beta, \chi) \in N_{\Lambda}(\psi(\bar{x}, \bar{y}))\right\}=\{(0,0,0)\},
$$

the second inclusion in (3.17) shows that $\left(A_{1}^{2}\right)$ is sufficient for this to hold. Furthermore, by (3.13) the second inclusion of (3.17) leads to an upper estimate of $N_{\mathrm{gph} S^{c}}$, which allows us via the coderivative definition (2.6) to recover the upper bound of $D^{*} S^{c}$ in the theorem. This implies, similarly to the proof of Theorem 3.1, that $S^{c}$ is Lipschitz-like around $(\bar{x}, \bar{y})$ under $\left(A_{2}^{2}\right)$.

As in the previous subsection, we arrive at the following sensitivity results for the OPCC value function (3.1) via C-multipliers.

TheOREm 3.6 (C-type sensitivity analysis for OPCC value functions). The following assertions hold for the value function $\mu^{c}$ in (3.1):

(i) Let the optimal solution map $S_{o}^{c}$ be inner semicontinuous at $(\bar{x}, \bar{y})$, and let $\left(A_{1}^{2}\right)$ hold at $(\bar{x}, \bar{y})$. Then we have the subdifferential upper estimate

$$
\partial \mu^{c}(\bar{x}) \subset\left\{\nabla_{x} L(\bar{x}, \bar{y}, \alpha, \beta, \gamma, \zeta) \mid(\alpha, \beta, \gamma, \zeta) \in \Lambda_{y}^{c c}(\bar{x}, \bar{y})\right\} .
$$

Copyright $@$ ㅇ by SIAM. Unauthorized reproduction of this article is prohibited. 
If in addition $\left(A_{2}^{2}\right)$ holds at $(\bar{x}, \bar{y})$, then $\mu^{c}$ is Lipschitz continuous around $\bar{x}$.

(ii) Assume that $S_{o}^{c}$ is inner semicompact at $\bar{x}$ and that $\left(A_{1}^{2}\right)$ holds at $(\bar{x}, \bar{y})$ for all $\bar{y} \in S_{o}^{c}(x)$. Then we have the subdifferential upper estimate

$$
\partial \mu^{c}(\bar{x}) \subset\left\{\nabla_{x} L(\bar{x}, \bar{y}, \alpha, \beta, \gamma, \zeta) \mid \bar{y} \in S_{o}^{c}(\bar{x}),(\alpha, \beta, \gamma, \zeta) \in \Lambda_{y}^{c c}(\bar{x}, \bar{y})\right\} .
$$

If in addition $\left(A_{2}^{2}\right)$ holds at $(\bar{x}, \bar{y})$ for $\bar{y} \in S_{o}^{c}(\bar{x})$, then $\mu^{c}$ is Lipschitzian around $\bar{x}$.

Proof. The proof follows from the results of Theorems 3.5 and 2.1.

Note that assertion (ii) of Theorem 3.6 can be found in [32] for $G(x, y)=y$ under a similar framework, but with a qualification condition corresponding to the replacement of $\Lambda^{c c}(\bar{x}, \bar{y}, 0)$ in $\left(A_{1}^{2}\right)$ by

$$
\begin{aligned}
\{(\alpha, \beta, \gamma, \zeta) \mid & \alpha \geq 0, \alpha^{\top} g(\bar{x}, \bar{y})=0, \\
& \gamma_{\eta}=0, \zeta_{\nu}=0, \\
& \forall i \in \theta, \exists t_{i} \in[0,1], r_{i} \in \mathbb{R} \text { s.t. } \gamma_{i}=r_{i} t_{i}, \zeta_{i}=r_{i}\left(1-t_{i}\right), \\
& \left.\nabla g(\bar{x}, \bar{y})^{\top} \alpha+\nabla h(\bar{x}, \bar{y})^{\top} \beta+\nabla G(\bar{x}, \bar{y})^{\top} \gamma+\nabla H(\bar{x}, \bar{y})^{\top} \zeta=0\right\} .
\end{aligned}
$$

The latter assumption is weaker than $\left(A_{1}^{2}\right)$, but in our device we simply need to check that the components of $\gamma$ and $\zeta$ are of the same sign on $\theta$ rather than constructing them as in the above set. Let us also mention that all the points made in Remark 3.3 can be restated here accordingly. In particular, $\left(A_{1}^{2}\right)$ can be substituted by the calmness of the set-valued mapping $\Psi$ from (3.9) with $\psi$ and $\Lambda$ given in (3.12). This is the case if the functions $g, h, G$, and $H$ are linear since $V_{i}(x, y)=\min \left\{G_{i}(x, y), H_{i}(x, y)\right\}$ is piecewise linear, provided $G_{i}$ and $H_{i}$ linear.

3.3. Sensitivity analysis via S-type multipliers. The S-type stationarity conditions in the context of OPCCs are the best one would want to have since these conditions are equivalent to the KKT-type optimality conditions whenever the OPCC is treated as an ordinary nonlinear optimization problem.

Having this in mind, we derive here a tighter upper bound for the basic subdifferential of the OPCC value function $\mu^{c}$. In order to obtain an upper bound for $\partial \mu^{c}$ containing $\Lambda_{y}^{c s}(\bar{x}, \bar{y})$ rather than $\Lambda_{y}^{c m}(\bar{x}, \bar{y})$ or $\Lambda_{y}^{c c}(\bar{x}, \bar{y})$, consider the index set

$$
I:=I(\bar{x}, \bar{y}):=\left\{i=1, \ldots, a \mid g_{i}(\bar{x}, \bar{y})<0\right\}
$$

and impose the following $S$-qualification condition

$$
\left.\begin{array}{rl}
\left(A_{1}^{3}\right) \quad \begin{array}{r}
\nabla L_{o}(\bar{x}, \bar{y}, \alpha, \beta, \gamma, \zeta) \\
\alpha_{I}=0, \gamma_{\nu}=0, \zeta_{\eta}
\end{array}=0
\end{array}\right\} \Longrightarrow \gamma_{\theta}=0, \zeta_{\theta}=0
$$

introduced by Ye [57] and later named in [59] as partial MPEC-LICQ (linear independence constraint qualification). This condition and another similar yet weaker one have also been used by Flegel, Kanzow, and Outrata [22] to recover the S-stationarity conditions for OPCCs from the M-ones. In the next theorem we obtain a new S-type upper bound for $\partial \mu^{c}$ by a similar methodology, i.e., going from the M-type bound provided above. Recall that assumption $\left(A_{1}^{1}\right)$ needed here is given in (3.5).

TheOREm 3.7 (S-type sensitivity analysis for OPCC value functions). The following assertions hold for the value function $\mu^{c}$ from (3.1):

(i) Let the optimal solution map $S_{o}^{c}$ be inner semicontinuous at $(\bar{x}, \bar{y})$, and let assumptions $\left(A_{1}^{1}\right)$ and $\left(A_{1}^{3}\right)$ be satisfied at $(\bar{x}, \bar{y})$. Then we have

$$
\partial \mu^{c}(\bar{x}) \subset\left\{\nabla_{x} L(\bar{x}, \bar{y}, \alpha, \beta, \gamma, \zeta) \mid(\alpha, \beta, \gamma, \zeta) \in \Lambda_{y}^{c s}(\bar{x}, \bar{y})\right\} .
$$

Copyright $@$ by SIAM. Unauthorized reproduction of this article is prohibited. 
(ii) Let $S_{o}^{c}$ be inner semicompact at $\bar{x}$ with $\left(A_{1}^{1}\right)$ and $\left(A_{1}^{3}\right)$ being satisfied at $(\bar{x}, \bar{y})$ for all $\bar{y} \in S_{o}^{c}(\bar{x})$. Then we have

$$
\partial \mu^{c}(\bar{x}) \subset\left\{\nabla_{x} L(\bar{x}, \bar{y}, \alpha, \beta, \gamma, \zeta) \mid \bar{y} \in S_{o}^{c}(\bar{x}),(\alpha, \beta, \gamma, \zeta) \in \Lambda_{y}^{c s}(\bar{x}, \bar{y})\right\} .
$$

Proof. We provide the proof only for assertion (i); the other case can be proved similarly. Assuming $\left(A_{1}^{1}\right)$ and the inner semicontinuity of $S_{o}^{c}$, we have the upper estimate of $\partial \mu^{c}$ from Theorem 3.2(i). Further, denote by $A(\bar{x}, \bar{y})$ (resp., $B(\bar{x}, \bar{y})$ ) the right-hand side of the inclusion in Theorem 3.2(i) (resp., Theorem 3.7(i)). It remains to show that $A(\bar{x}, \bar{y})=B(\bar{x}, \bar{y})$ under the S-qualification condition $\left(A_{1}^{3}\right)$.

We obviously have $A(\bar{x}, \bar{y}) \supset B(\bar{x}, \bar{y})$. To justify the opposite inclusion, pick any $a(\alpha, \beta, \gamma, \zeta) \in A(\bar{x}, \bar{y})$ and search for $b\left(\alpha^{o}, \beta^{o}, \gamma^{o}, \zeta^{o}\right) \in B(\bar{x}, \bar{y})$ such that $a(\alpha, \beta, \gamma, \zeta)=$ $b\left(\alpha^{o}, \beta^{o}, \gamma^{o}, \zeta^{o}\right)$. If the latter equality were to hold, we would get

$$
\left\{\begin{array}{l}
\nabla L_{o}\left(\bar{x}, \bar{y}, \alpha-\alpha^{o}, \beta-\beta^{o}, \gamma-\gamma^{o}, \zeta-\zeta^{o}\right)=0 \\
\alpha_{I}^{o}-\alpha_{I}^{o}=0, \gamma_{\nu}-\gamma_{\nu}^{o}=0, \zeta_{\eta}-\zeta_{\eta}^{o}=0
\end{array}\right.
$$

Thus it follows from $\left(A_{1}^{3}\right)$ that $\gamma_{\theta}^{o}=\gamma_{\theta}$ and $\zeta_{\theta}^{o}=\zeta_{\theta}$. To conclude the proof, choose $\alpha^{o}:=\alpha, \beta^{o}:=\beta, \gamma_{\theta^{c}}^{o}:=\gamma_{\theta^{c}}$, and $\zeta_{\theta^{c}}^{o}:=\zeta_{\theta^{c}}$ with $\theta^{c}:=\{i=1, \ldots, d\} \backslash \theta$.

Observe from the proof above that it can be repeated by using the C-type upper bound in subsection 3.2 instead of the M-one. This shows that under the assumption $\left(A_{1}^{3}\right)$ all the S-type, M-type, and C-type upper bounds for $\partial \mu^{c}$ are the same.

Either replacing $\left(A_{1}^{1}\right)$ by $\left(A_{3}^{1}\right)$ or adding $\left(A_{2}^{1}\right)$ to the assumptions of Theorem 3.7, we get the local Lipschitz continuity of $\mu^{c}$. On the other hand, replacing $\left(A_{1}^{3}\right)$ by the stronger well-known MPEC-LICQ formulated as

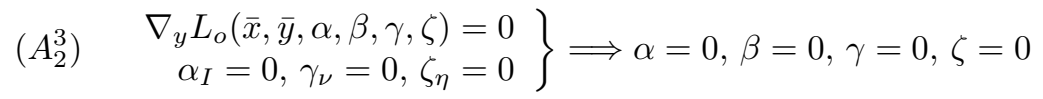

ensures even more than the Lipschitz continuity of $\mu^{c}$; namely, its strict differentiability as stated in the next corollary.

Corollary 3.8 (strict differentiability of OPCC value functions). Assume that $S_{o}^{c}$ is inner semicontinuous at the point $(\bar{x}, \bar{y})$, where $\left(A_{2}^{3}\right)$ is also satisfied. Then the value function $\mu^{c}$ is strictly differentiable at $\bar{x}$ with

$$
\nabla \mu^{c}(\bar{x})=\nabla_{x} L(\bar{x}, \bar{y}, \alpha, \beta, \gamma, \zeta),
$$

where $(\alpha, \beta, \gamma, \zeta)$ is the unique multiplier of the set $\Lambda_{y}^{c s}(\bar{x}, \bar{y})$.

Proof. We can see that the set on the right-hand side of the inclusion in Theorem 3.7(i) is a singleton; hence $\partial \mu^{c}(\bar{x})$ is a singleton as well. Since the value function $\mu^{c}$ is surely locally Lipschitzian around $\bar{x}$ under the MPEC-LICQ (3.19), the latter uniqueness ensures its strict differentiability at this point; see section 2 .

In case of (ii) we additionally need $S_{o}^{c}(\bar{x})$ to be a singleton to ensure the strict differentiability of $\mu^{c}$ at $\bar{x}$. The latter corresponds to the framework provided by $\mathrm{Hu}$ and Ralph [29], and hence it shows that the assumptions imposed in [29] imply the inner semicontinuity of the set-valued mapping $S_{o}^{c}$ at the solution point. Note also that assertion (ii) of Theorem 3.7 closely relates to the corresponding result of [32] obtained in a particular case from a different perspective.

4. Sensitivity analysis of OPEC value functions. This section is devoted to the study of the following value function of a parametric optimization problem with generalized equation constraints (OPEC):

$$
\mu^{e}(x):=\min _{y}\left\{F(x, y) \mid y \in S^{e}(x):=\left\{y \mid 0 \in h(x, y)+N_{K(x)}(y)\right\}\right\},
$$


where $F: \mathbb{R}^{n} \times \mathbb{R}^{m} \rightarrow \mathbb{R}, h: \mathbb{R}^{n} \times \mathbb{R}^{m} \rightarrow \mathbb{R}^{p}$ are continuously differentiable functions, and $K$ denotes a set-valued mapping (moving set) defined by (1.3) with $g$ also continuously differentiable and $g(x, \cdot)$ convex for all $x \in \mathbb{R}^{n}$. If we assume that the lower-level regularity condition

$$
\left[\nabla_{y} g_{i}(\bar{x}, \bar{y})^{\top} \beta=0, \beta \geq 0, \beta^{\top} g(\bar{x}, \bar{y})=0\right] \Longrightarrow \beta=0
$$

holds at $(x, y) \in \operatorname{gph} K$, then the normal cone to $K(x)$ at $y$ reduces to

$$
N_{K(x)}(y)=\left\{\nabla_{y} g(x, y)^{\top} u \mid u \geq 0, u^{\top} g(x, y)=0\right\} .
$$

Inserting this expression in (4.1) gives a particular case of (3.1). However, it appears to be of independent interest to study sensitivity properties of $\mu^{e}(4.1)$ in its actual form, since this way we obtain different estimates for the limiting subdifferential of the two-level value function $\varphi_{o}$ from (1.4), which is of our main focus in this paper. This issue will be comprehensively discussed in the next section.

A detailed study of the robust Lipschitzian stability of $S^{e}$ based on coderivative analysis has been carried out by Mordukhovich and Outrata [44]. Note that the work in [44] heavily relies on an estimate of the coderivative of the normal cone mapping $(x, y) \rightrightarrows N_{K(x)}(y)$ given therein. Before introducing the rules to be used here (which emerged from [44]), some notation is necessary to simplify the presentation. Define $\mathcal{L}(x, y, u):=h(x, y)+\nabla_{y} g(x, y)^{\top} u$ and consider the set of lower-level Lagrange multipliers $\Lambda(\bar{x}, \bar{y}):=\left\{u \mid \mathcal{L}(\bar{x}, \bar{y}, u)=0, u \geq 0, u^{\top} g(\bar{x}, \bar{y})=0\right\}$.

Similarly to the previous section, we partition the indices of the functions involved in the complementarity system in (4.3) as follows:

$$
\begin{aligned}
& \eta=\eta(\bar{x}, \bar{y}, \bar{u}):=\left\{i=1, \ldots, p \mid \bar{u}_{i}=0, g_{i}(\bar{x}, \bar{y})<0\right\}, \\
& \theta=\theta(\bar{x}, \bar{y}, \bar{u}):=\left\{i=1, \ldots, p \mid \bar{u}_{i}=0, g_{i}(\bar{x}, \bar{y})=0\right\}, \\
& \nu=\nu(\bar{x}, \bar{y}, \bar{u}):=\left\{i=1, \ldots, p \mid \bar{u}_{i}>0, g_{i}(\bar{x}, \bar{y})=0\right\} .
\end{aligned}
$$

Consider also the system of relationships that play an important role in what follows:

$$
\begin{aligned}
v+\nabla g(\bar{x}, \bar{y})^{\top} \beta+\nabla_{x, y} \mathcal{L}(\bar{x}, \bar{y}, \bar{u})^{\top} \gamma & =0, \\
\nabla_{y} g_{\nu}(\bar{x}, \bar{y}) \gamma=0, \beta_{\eta} & =0, \\
\forall i \in \theta,\left(\beta_{i}>0 \wedge \nabla_{y} g_{i}(\bar{x}, \bar{y}) \gamma>0\right) \vee \beta_{i}\left(\nabla_{y} g_{i}(\bar{x}, \bar{y}) \gamma\right) & =0 .
\end{aligned}
$$

The corresponding set of multipliers, which are of a special M-type, are defined by

$$
\Lambda^{e m}(\bar{x}, \bar{y}, \bar{u}, v):=\{(\beta, \gamma) \mid(4.5)-(4.7) \text { hold }\} .
$$

Similarly to section 3 , we further define $\Lambda_{y}^{e m}(\bar{x}, \bar{y}, \bar{u}, v)$ as $v \in \mathbb{R}^{m}$ by replacing (4.5) with $v+\nabla_{y} g(\bar{x}, \bar{y})^{\top} \beta+\nabla_{y} \mathcal{L}(\bar{x}, \bar{y}, \bar{u})^{\top} \gamma=0$ and then setting $\Lambda_{y}^{e m}(\bar{x}, \bar{y}, \bar{u}):=$ $\Lambda_{y}^{e m}\left(\bar{x}, \bar{y}, \bar{u}, \nabla_{y} F(\bar{x}, \bar{y})\right)$. The following EM-qualification conditions deduced from [44] can be formulated as

$$
\begin{array}{ll}
\left(A_{1}^{4}\right) & {\left[\bar{u} \in \Lambda(\bar{x}, \bar{y}), \nabla g(\bar{x}, \bar{y})^{\top} \beta=0, \beta_{\eta}=0\right] \Longrightarrow \beta=0,} \\
\left(A_{2}^{4}\right) & {\left[\bar{u} \in \Lambda(\bar{x}, \bar{y}),(\beta, \gamma) \in \Lambda^{e m}(\bar{x}, \bar{y}, \bar{u}, 0)\right] \Longrightarrow \beta=0, \gamma=0,} \\
\left(A_{3}^{4}\right) & {\left[\bar{u} \in \Lambda(\bar{x}, \bar{y}),(\beta, \gamma) \in \Lambda_{y}^{e m}(\bar{x}, \bar{y}, \bar{u}, 0)\right] \Longrightarrow \nabla_{x} g(\bar{x}, \bar{y})^{\top} \beta+\nabla_{x} \mathcal{L}(\bar{x}, \bar{y}, \bar{u})^{\top} \gamma=0,} \\
\left(A_{4}^{4}\right) & {\left[\bar{u} \in \Lambda(\bar{x}, \bar{y}),(\beta, \gamma) \in \Lambda_{y}^{e m}(\bar{x}, \bar{y}, \bar{u}, 0)\right] \Longrightarrow \beta=0, \gamma=0 .}
\end{array}
$$

Note that in $\left(A_{1}^{4}\right)$ the index set $\eta$ depends on $\bar{u}$ by means of its definition in (4.4). It is easy to observe the relationships $\left(A_{3}^{4}\right) \Longleftarrow\left(A_{4}^{4}\right) \Longrightarrow\left(A_{2}^{4}\right),\left(A_{1}^{4}\right)$. 
We are now ready to establish the main result of this section, where $S_{o}^{e}$ denotes the solution map to the parametric optimization problem related to (4.1) given by

$$
S_{o}^{e}(x):=\left\{y \in S^{e}(x) \mid F(x, y)-\mu^{e}(x) \leq 0\right\} .
$$

THEOREM 4.1 (M-type sensitivity analysis for OPEC value functions). The following assertions hold for the value function $\mu^{e}$ from (4.1):

(i) Let the optimal solution map $S_{o}^{e}$ be inner semicontinuous at the point $(\bar{x}, \bar{y})$, where the lower-level regularity (4.2) and the conditions $\left(A_{1}^{4}\right)-\left(A_{2}^{4}\right)$ are satisfied. Then we have the subdifferential estimate

$$
\partial \mu^{e}(\bar{x}) \subset \bigcup_{\bar{u} \in \Lambda(\bar{x}, \bar{y})} \bigcup_{(\beta, \gamma) \in \Lambda_{y}^{\text {em }}(\bar{x}, \bar{y}, \bar{u})}\left\{\nabla_{x} F(\bar{x}, \bar{y})+\nabla_{x} g(\bar{x}, \bar{y})^{\top} \beta+\nabla_{x} \mathcal{L}(\bar{x}, \bar{y}, \bar{u})^{\top} \gamma\right\} .
$$

If in addition $\left(A_{3}^{4}\right)$ holds at $(\bar{x}, \bar{y})$, then $\mu^{e}$ is Lipschitz continuous around $\bar{x}$.

(ii) Let $S_{o}^{e}$ be inner semicompact at $\bar{x}$, and let $\left(A_{1}^{4}\right)-\left(A_{2}^{4}\right)$ and lower-level regularity (4.2) be satisfied at $(\bar{x}, \bar{y})$ for all $\bar{y} \in S_{o}^{e}(\bar{x})$. Then we have the subdifferential estimate

$$
\begin{aligned}
\partial \mu^{e}(\bar{x}) \subset \bigcup_{\bar{y} \in S_{o}(\bar{x})} \bigcup_{\bar{u} \in \Lambda(\bar{x}, \bar{y})} \bigcup_{(\beta, \gamma) \in \Lambda_{y}^{e m}(\bar{x}, \bar{y}, \bar{u})}\left\{\nabla_{x} F(\bar{x}, \bar{y})\right. & +\nabla_{x} g(\bar{x}, \bar{y})^{\top} \beta \\
& \left.+\nabla_{x} \mathcal{L}(\bar{x}, \bar{y}, \bar{u})^{\top} \gamma\right\} .
\end{aligned}
$$

If $\left(A_{3}^{4}\right)$ also holds at $(\bar{x}, \bar{y})$ as $\bar{y} \in S_{o}^{e}(\bar{x})$, then $\mu^{e}$ is Lipschitz continuous around $\bar{x}$.

Proof. We justify only assertion (i); the one in (ii) can be proved similarly. Since $F$ is continuously differentiable and $S_{o}^{e}$ is inner semicontinuous, it follows from Theorem 2.1(i) that

$$
\partial \mu^{e}(\bar{x}) \subset \nabla_{x} F(\bar{x}, \bar{y})+D^{*} S^{e}(\bar{x}, \bar{y})\left(\nabla_{y} F(\bar{x}, \bar{y})\right) .
$$

Further applying [44, Theorem 4.3] to the solution map $S^{e}$ and taking into account that the $E M$-qualification conditions $\left(A_{1}^{4}\right)-\left(A_{2}^{4}\right)$ together with the lower-level regularity (4.2) are satisfied, we get the coderivative estimate

$$
D^{*} S^{e}(\bar{x}, \bar{y})\left(\nabla_{y} F(\bar{x}, \bar{y})\right) \subset \bigcup_{\bar{u} \in \Lambda(\bar{x}, \bar{y})} \bigcup_{(\beta, \gamma) \in \Lambda_{y}^{e m}(\bar{x}, \bar{y}, \bar{u})}\left\{\nabla_{x} g(\bar{x}, \bar{y})^{\top} \beta+\nabla_{x} \mathcal{L}(\bar{x}, \bar{y}, \bar{u})^{\top} \gamma\right\} .
$$

Then the upper estimate of the basic subdifferential of $\mu^{e}$ in (i) follows by inserting the latter inclusion in (4.8). The local Lipschitz continuity of $\mu^{e}$ around $\bar{x}$ also follows from Theorem 2.1(i) by recalling (see [44, Theorem 5.1]) that $S^{e}$ is Lipschitz-like around $(\bar{x}, \bar{y})$ if we add $\left(A_{3}^{4}\right)$ to the previous assumptions.

To the best of our knowledge, the first result in the direction of Theorem 4.1(ii) goes back to Lucet and Ye [32], where a similar subdifferential estimate was obtained under a growth hypothesis (similar to the uniform boundedness of $S_{o}^{e}$; see (2.7)) for a particular case of the problem under consideration. Note, however, that their result deals only with the case where $K$ is independent of $x$. Assertion (i) of Theorem 4.1 clearly provides a tighter subdifferential upper bound under the inner semicontinuity assumption. We also mention the work by Mordukhovich, Nam, and Yen [43] in the framework where the regular and limiting subdifferentials of $\mu^{e}$ are estimated in the case of $S^{e}(x):=\{y \mid 0 \in h(x, y)+Q(x, y)\}$ in (4.1) with a general set-valued mapping $Q(x, y)$ not specified to our setting $Q(x, y):=N_{K(x)}(y)$.

Remark 4.2 (sensitivity analysis for OPEC value functions under the calmness condition). Following Mordukhovich and Outrata [44], the qualification condition 
$\left(A_{1}^{4}\right)$ in Theorem 4.1 can be replaced by the weaker calmness property of the following set-valued mapping at $(0, \bar{x}, \bar{y}, \bar{u})$ for all $\bar{u} \in \Lambda(\bar{x}, \bar{y})$ :

$$
M(v):=\left\{(x, y, u) \mid[g(x, y), u]+v \in \operatorname{gph} N_{\mathbb{R}_{-}^{p}}\right\} .
$$

Similarly, condition $\left(A_{2}^{4}\right)$ can be replaced by the calmness property of the mapping

$$
P(z, v):=\{(x, y, u) \mid \mathcal{L}(x, y, u)+z=0\} \cap M(v)
$$

at $(0,0, \bar{x}, \bar{y}, \bar{u})$ for all $\bar{u} \in \Lambda(\bar{x}, \bar{y})$. Both calmness assumptions are automatic when the mappings $g$ and $(x, y) \mapsto \nabla_{y} f(x, y)$ are linear. It is pointed out to us by René Henrion that it follows from the combination of Proposition 4.1 in the paper by Henrion, Outrata, and Surowiec [26] with a recent calmness result by Lu [31] that the calmness property of $M$ holds if the parametric inequality system $g(x, y) \leq p$ satisfies the constant rank constraint qualification at the point of interest when $p=0$.

5. Sensitivity analysis of two-level value functions. The main goal of this section is to conduct a local sensitivity analysis of the two-level optimal value function

$$
\varphi_{o}(x):=\min _{y}\{F(x, y) \mid y \in S(x)\}
$$

where the lower-level optimal solution map $S$ is defined in (1.5). In the next subsection we explore all three approaches $(\mathrm{M}, \mathrm{C}$, and $\mathrm{S})$ to this issue discussed in sections 3 and 4 . The lower-level value function representation of the mapping $S$ from (1.5) is considered in subsection 5.2.

5.1. OPCC and OPEC approaches. From now on in this subsection we assume that the lower-level problem (1.2) with $K(x)$ given by (1.3) is convex; i.e., the functions $f(x,$.$) and g(x,$.$) are convex for all x \in X$. Most of the notation below is either taken from section 4 or closely related to it. To be more precise, from now on the lower-level Lagrangian-type function and multipliers set are defined by

$$
\begin{aligned}
& \mathcal{L}(x, y, u):=\nabla_{y} f(x, y)+\nabla_{y} g(x, y)^{\top} u \text { and } \\
& \Lambda(\bar{x}, \bar{y}):=\left\{u \mid \mathcal{L}(\bar{x}, \bar{y}, u)=0, u \geq 0, g(\bar{x}, \bar{y}) \leq 0, u^{\top} g(\bar{x}, \bar{y})=0\right\}
\end{aligned}
$$

respectively, while the index sets $\eta, \theta$, and $\nu$ are given in (4.4). The next lemma involving $\mathcal{L}$ is useful in what follows.

Lemma 5.1 (representation of the two-level value function). Let $x \in X$ and assume that $f(x,$.$) and g(x,$.$) are convex. Furthermore, we suppose that the lower-$ level regularity $(4.2)$ is satisfied at $(x, y)$ for all $y \in S(x)$. Then we have

$$
\varphi_{o}(x)=\min _{y, u}\left\{F(x, y) \mid \mathcal{L}(x, y, u)=0, u \geq 0, g(x, y) \leq 0, u^{\top} g(x, y)=0\right\} .
$$

Proof. Fix $\bar{x} \in X$ and let the point $\bar{y}$ be a global optimal solution to the problem $\min _{y}\{F(\bar{x}, y) \mid y \in S(\bar{x})\}$. Then we have the relationships

$$
\begin{aligned}
\varphi_{o}(\bar{x})= & F(\bar{x}, \bar{y}) \\
\leq & F(\bar{x}, y) \forall y \in S(\bar{x}), \\
\leq & F(\bar{x}, y) \forall y \text { with } 0 \in \nabla_{y} f(\bar{x}, y)+N_{K(\bar{x})}(y) \\
& (\text { by convexity of } f(\bar{x}, .) \text { and } g(\bar{x}, .)), \\
\leq & F(\bar{x}, y) \forall(y, u) \text { with } \mathcal{L}(\bar{x}, y, u)=0, u \geq 0, g(\bar{x}, y) \leq 0, u^{\top} g(\bar{x}, y)=0,
\end{aligned}
$$

Copyright (C) by SIAM. Unauthorized reproduction of this article is prohibited. 
where the last inequality is due to the normal cone representation (4.3) by taking into account that the lower-level regularity (4.2) holds at all $(\bar{x}, y)$ with $y \in S(\bar{x})$.

Having this transformation of the two-level value function $\varphi_{o}$, at least two observations can be made. First, we note that for each $x \in X$ the value of $\varphi_{o}(x)$ is obtained from a global solution to the parametric problem

$$
\min _{y, u}\left\{F(x, y) \mid \mathcal{L}(x, y, u)=0, u \geq 0, g(x, y) \leq 0, u^{\top} g(x, y)=0\right\} .
$$

Thus the major difficulty arising when establishing the link between local solutions of the auxiliary problem (P) and its KKT reformulation (see [10] for details) does not appear here. Second, the presence of the complementarity constraints $u \geq 0, g(x, y) \leq$ $0, u^{\top} g(x, y)=0$ in (5.4) leads to the violation of the MFCQ, while the results of section 3 can be applied. To proceed, consider the optimal solution map of problem (5.4) given by

$$
S_{o}^{h}(x):=\left\{(y, u) \mid u \in \Lambda(x, y), \quad F(x, y) \leq \varphi_{o}(x)\right\} .
$$

Now we establish M-type sensitivity results for the two-level value function $\varphi_{o}$, which are crucial in the paper. In the vein of the rules in (3.5), the following modified $M$-qualification conditions are used in the next theorem:

$$
\begin{array}{ll}
\left(A_{1}^{m}\right) & (\beta, \gamma) \in \Lambda^{e m}(\bar{x}, \bar{y}, \bar{u}, 0) \Longrightarrow \beta=0, \gamma=0, \\
\left(A_{2}^{m}\right) & (\beta, \gamma) \in \Lambda_{y}^{e m}(\bar{x}, \bar{y}, \bar{u}, 0) \Longrightarrow \nabla_{x} g(\bar{x}, \bar{y})^{\top} \beta+\nabla_{x} \mathcal{L}(\bar{x}, \bar{y}, \bar{u})^{\top} \gamma=0, \\
\left(A_{3}^{m}\right) & (\beta, \gamma) \in \Lambda_{y}^{e m}(\bar{x}, \bar{y}, \bar{u}, 0) \Longrightarrow \beta=0, \gamma=0,
\end{array}
$$

where the multiplier sets $\Lambda^{e m}(\bar{x}, \bar{y}, \bar{u}, 0), \Lambda_{y}^{e m}(\bar{x}, \bar{y}, \bar{u}, 0)$, and $\Lambda_{y}^{e m}(\bar{x}, \bar{y}, \bar{u})$ are exactly the ones defined in section 4 while setting $h(x, y):=\nabla_{y} f(x, y)$.

Theorem 5.2 (M-type sensitivity analysis for the two-level value function). Let the lower-level problem (1.2) be convex, and assume that the lower-level regularity (4.2) holds at all $(\bar{x}, y)$ with $y \in S(\bar{x})$. Then the following assertions are satisfied:

(i) If the optimal solution map $S_{o}^{h}$ in $(5.5)$ is inner semicontinuous at $(\bar{x}, \bar{y}, \bar{u})$ and if $\left(A_{1}^{m}\right)$ holds at this point, then the limiting subdifferential of $\varphi_{o}$ is estimated by

$$
\partial \varphi_{o}(\bar{x}) \subset \bigcup_{(\beta, \gamma) \in \Lambda_{y}^{e m}(\bar{x}, \bar{y}, \bar{u})}\left\{\nabla_{x} F(\bar{x}, \bar{y})+\nabla_{x} g(\bar{x}, \bar{y})^{\top} \beta+\nabla_{x} \mathcal{L}(\bar{x}, \bar{y}, \bar{u})^{\top} \gamma\right\} .
$$

Furthermore, $\varphi_{o}$ is Lipschitz continuous around $\bar{x}$ if $\left(A_{2}^{m}\right)$ is also satisfied at $(\bar{x}, \bar{y}, \bar{u})$.

(ii) Let the set-valued mapping $S_{o}^{h}$ in (5.5) be inner semicompact at $\bar{x}$, and let condition $\left(A_{1}^{m}\right)$ hold at $(\bar{x}, \bar{y}, \bar{u})$ for all $(\bar{y}, \bar{u}) \in S_{o}^{h}(\bar{x})$. Then

$$
\partial \varphi_{o}(\bar{x}) \subset \bigcup_{(\bar{y}, \bar{u}) \in S_{o}^{h}(\bar{x})} \bigcup_{(\beta, \gamma) \in \Lambda_{y}^{e m}(\bar{x}, \bar{y}, \bar{u})}\left\{\nabla_{x} F(\bar{x}, \bar{y})+\nabla_{x} g(\bar{x}, \bar{y})^{\top} \beta+\nabla_{x} \mathcal{L}(\bar{x}, \bar{y}, \bar{u})^{\top} \gamma\right\} .
$$

If in addition condition $\left(A_{2}^{m}\right)$ is satisfied at $(\bar{x}, \bar{y}, \bar{u})$ for all $(\bar{y}, \bar{u}) \in S_{o}^{h}(\bar{x})$, then $\varphi_{o}$ is Lipschitz continuous around $\bar{x}$.

Proof. We give the proof only for (i) since (ii) can be obtained similarly. By setting $y:=(y, u)$ in the framework of Theorem 3.2, we just need to specify the multiplier sets therein to our setting. It follows from Lemma 5.1 that

$$
\begin{aligned}
& \varphi_{o}(x)=\min _{y, u}\{F(x, y) \mid h(x, y, u)=0, \\
&\left.G(x, y, u) \geq 0, H(x, y, u) \geq 0, G(x, y, u)^{\top} H(x, y, u)=0\right\},
\end{aligned}
$$

Copyright $@$ by SIAM. Unauthorized reproduction of this article is prohibited. 
where $h(x, y, u):=\mathcal{L}(x, y, u), G(x, y, u):=u$, and $H(x, y, u):=-g(x, y)$. Thus we have from section 3 , while using the notational conventions of section 4 in the framework of (5.7), that

$$
\begin{aligned}
\Lambda^{c m}\left(\bar{x}, \bar{y}, \bar{u}, v_{1}, v_{2}, v_{3}\right)=\{ & (\beta, \gamma, \zeta) \mid \zeta_{\nu}=0, \beta_{\eta}=0, \\
& \left(\zeta_{i}<0, \beta_{i}<0\right) \vee\left(\beta_{i} \zeta_{i}=0\right) \forall i \in \theta, \\
& v_{1}+\nabla_{x} \mathcal{L}(\bar{x}, \bar{y}, \bar{u})^{\top} \gamma-\nabla_{x} g(\bar{x}, \bar{y})^{\top} \beta=0, \\
& v_{2}+\nabla_{y} \mathcal{L}(\bar{x}, \bar{y}, \bar{u})^{\top} \gamma-\nabla_{y} g(\bar{x}, \bar{y})^{\top} \beta=0, \\
& \left.v_{3}+\nabla_{y} g(\bar{x}, \bar{y}) \gamma+\zeta=0\right\} .
\end{aligned}
$$

Now note that from (5.8), we get equality $\zeta=-\nabla_{y} g(\bar{x}, \bar{y}) \gamma$ by setting $v_{3}:=0$ in $v_{3}+\nabla_{y} g(\bar{x}, \bar{y}) \gamma+\zeta=0$. Thus

$$
\begin{aligned}
\Lambda^{c m}(\bar{x}, \bar{y}, \bar{u}, 0)=\{ & \left(\beta, \gamma,-\nabla_{y} g(\bar{x}, \bar{y}) \gamma\right) \mid \nabla_{y} g_{\nu}(\bar{x}, \bar{y}) \gamma=0, \beta_{\eta}=0, \\
& \left(\nabla_{y} g_{i}(\bar{x}, \bar{y}) \gamma>0, \beta_{i}<0\right) \vee \beta_{i}\left(\nabla_{y} g_{i}(\bar{x}, \bar{y}) \gamma\right)=0 \forall i \in \theta, \\
& \left.\sum_{l=1}^{m} \gamma_{l} \nabla_{x, y} \mathcal{L}_{l}(\bar{x}, \bar{y}, \bar{u})-\sum_{i=1}^{p} \beta_{i} \nabla g_{i}(\bar{x}, \bar{y})=0\right\} \\
= & \left\{\left(\beta, \gamma,-\nabla_{y} g(\bar{x}, \bar{y}) \gamma\right) \mid(-\beta, \gamma) \in \Lambda^{e m}(\bar{x}, \bar{y}, \bar{u}, 0)\right\} .
\end{aligned}
$$

It is easy to observe from the latter equality that condition $\left(A_{1}^{m}\right)$ is sufficient for the following implication to hold:

$$
(\beta, \gamma, \zeta) \in \Lambda^{c m}(\bar{x}, \bar{y}, \bar{u}, 0) \Longrightarrow \beta=0, \gamma=0, \zeta=0 .
$$

Employing (5.9), we have from Theorem 3.2(i) that the limiting subdifferential of the two-level value function $\varphi_{o}$ (5.1) can be estimated by

$$
\partial \varphi_{o}(\bar{x}) \subset \bigcup_{(\beta, \gamma, \zeta) \in \Lambda_{y}^{c m}(\bar{x}, \bar{y}, \bar{u})}\left\{\nabla_{x} F(\bar{x}, \bar{y})-\sum_{i=1}^{p} \nabla_{x} g(\bar{x}, \bar{y})^{\top} \beta+\nabla_{x} \mathcal{L}(\bar{x}, \bar{y}, \bar{u})^{\top} \gamma\right\}
$$

where, similarly to the above, the multiplier set $\Lambda_{y}^{c m}(\bar{x}, \bar{y}, \bar{u})$ can be written as

$$
\begin{aligned}
\Lambda_{y}^{c m}(\bar{x}, \bar{y}, \bar{u}): & :=\Lambda_{y}^{c m}\left(\bar{x}, \bar{y}, \bar{u}, \nabla_{y} F(\bar{x}, \bar{y}), 0\right) \\
& =\left\{\left(\beta, \gamma,-\nabla_{y} g(\bar{x}, \bar{y}) \gamma\right) \mid(-\beta, \gamma) \in \Lambda_{y}^{e m}(\bar{x}, \bar{y}, \bar{u})\right\} .
\end{aligned}
$$

Thus $(\beta, \gamma, \zeta) \in \Lambda_{y}^{c m}(\bar{x}, \bar{y}, \bar{u})$ if and only if we have the relationships $\zeta=-\nabla_{y} g(\bar{x}, \bar{y}) \gamma$ and $(-\beta, \gamma) \in \Lambda_{y}^{e m}(\bar{x}, \bar{y}, \bar{u})$. This implies the inclusion in (i) of the theorem by noting that the term $\nabla_{x} F(\bar{x}, \bar{y})-\nabla_{x} g(\bar{x}, \bar{y})^{\top} \beta+\nabla_{x} \mathcal{L}(\bar{x}, \bar{y}, \bar{u})^{\top} \gamma$ does not depend on the multiplier $\zeta$. Proceeding further as above, we get that $\left(A_{2}^{m}\right)$ yields

$$
(\beta, \gamma, \zeta) \in \Lambda^{c m}(\bar{x}, \bar{y}, \bar{u}, 0) \Longrightarrow-\sum_{i=1}^{p} \beta_{i} \nabla_{x} g_{i}(\bar{x}, \bar{y})+\sum_{l=1}^{m} \gamma_{l} \nabla_{x} \mathcal{L}_{l}(\bar{x}, \bar{y}, \bar{u})=0,
$$

which implies in turn that the two-level value function $\varphi_{o}$ is Lipschitz continuous around $\bar{x}$ by applying Theorem 3.2(i) via the reformulation of $\varphi_{o}$ in (5.7). In the same way condition $\left(A_{3}^{m}\right)$ ensures the validity of the counterpart of $\left(A_{3}^{1}\right)$ in (3.5).

Copyright $@$ ㅇ by SIAM. Unauthorized reproduction of this article is prohibited. 
We can similarly consider the $C$-type multiplier sets $\Lambda^{e c}(\bar{x}, \bar{y}, \bar{u}, 0), \Lambda_{y}^{e c}(\bar{x}, \bar{y}, \bar{u}, 0)$, and $\Lambda_{y}^{e c}(\bar{x}, \bar{y}, \bar{u})$ by replacing condition (4.7) in $\Lambda^{e m}(\bar{x}, \bar{y}, \bar{u}, 0), \Lambda^{e m}(\bar{x}, \bar{y}, \bar{u}, 0)$, and $\Lambda_{y}^{e m}(\bar{x}, \bar{y}, \bar{u})$ with

$$
\forall i \in \theta, \beta_{i} \sum_{l=1}^{m} \gamma_{l} \nabla_{y_{l}} g_{i}(\bar{x}, \bar{y}) \geq 0
$$

Then the upper bounds of the limiting subdifferential via C-type multipliers and the local Lipschitz continuity of the two-level value function $\varphi_{o}$ under the C-type qualifications can be derived as in Theorem 5.2 with $\Lambda^{e m}(\bar{x}, \bar{y}, \bar{u}, 0), \Lambda_{y}^{e m}(\bar{x}, \bar{y}, \bar{u}, 0)$, and $\Lambda_{y}^{e m}(\bar{x}, \bar{y}, \bar{u})$ replaced by $\Lambda^{e c}(\bar{x}, \bar{y}, \bar{u}, 0), \Lambda_{y}^{e c}(\bar{x}, \bar{y}, \bar{u}, 0)$, and $\Lambda_{y}^{e c}(\bar{x}, \bar{y}, \bar{u})$, respectively. In this way we use, similarly to (5.6), the modified $C$-qualification conditions

$$
\begin{aligned}
& \left(A_{1}^{c}\right) \quad(\beta, \gamma) \in \Lambda^{e c}(\bar{x}, \bar{y}, \bar{u}, 0) \Longrightarrow \beta=0, \gamma=0, \\
& \left(A_{2}^{c}\right) \quad(\beta, \gamma) \in \Lambda_{y}^{e c}(\bar{x}, \bar{y}, \bar{u}, 0) \Longrightarrow \nabla_{x} g(\bar{x}, \bar{y})^{\top} \beta+\nabla_{x} \mathcal{L}(\bar{x}, \bar{y}, \bar{u})^{\top}=0, \\
& \left(A_{3}^{c}\right) \quad(\beta, \gamma) \in \Lambda_{y}^{e c}(\bar{x}, \bar{y}, \bar{u}, 0) \Longrightarrow \beta=0, \gamma=0 .
\end{aligned}
$$

ThEOREM 5.3 (C-type sensitivity analysis of the two-level value function). Let the lower-level problem (1.2) be convex, and let the lower-level regularity (4.2) hold at $(\bar{x}, y)$ for all $y \in S(\bar{x})$. Then the following assertions are satisfied:

(i) Assume that the optimal solution map $S_{o}^{h}$ in (5.5) is inner semicontinuous at $(\bar{x}, \bar{y}, \bar{u})$, where condition $\left(A_{1}^{c}\right)$ holds. Then we have

$$
\partial \varphi_{o}(\bar{x}) \subset \bigcup_{(\beta, \gamma) \in \Lambda_{y}^{e c}(\bar{x}, \bar{y}, \bar{u})}\left\{\nabla_{x} F(\bar{x}, \bar{y})+\nabla_{x} g(\bar{x}, \bar{y})^{\top} \beta+\nabla_{x} \mathcal{L}(\bar{x}, \bar{y}, \bar{u})^{\top} \gamma\right\} .
$$

Furthermore, $\varphi_{o}$ is Lipschitz continuous around $\bar{x}$ if $\left(A_{2}^{c}\right)$ is also satisfied at $(\bar{x}, \bar{y}, \bar{u})$.

(ii) Let $S_{o}^{h}$ be inner semicompact at $\bar{x}$, and let condition $\left(A_{1}^{c}\right)$ hold at $(\bar{x}, \bar{y}, \bar{u})$ for all $(\bar{y}, \bar{u}) \in S_{o}^{h}(\bar{x})$. Then we have

$$
\partial \varphi_{o}(\bar{x}) \subset \bigcup_{(\bar{y}, \bar{u}) \in S_{o}^{h}(\bar{x})} \bigcup_{(\beta, \gamma) \in \Lambda_{y}^{e c}(\bar{x}, \bar{y}, \bar{u})}\left\{\nabla_{x} F(\bar{x}, \bar{y})+\nabla_{x} g(\bar{x}, \bar{y})^{\top} \beta+\nabla_{x} \mathcal{L}(\bar{x}, \bar{y}, \bar{u})^{\top} \gamma\right\} .
$$

If condition $\left(A_{2}^{c}\right)$ is also satisfied at $(\bar{x}, \bar{y}, \bar{u})$ for all $(\bar{y}, \bar{u}) \in S_{o}^{h}(\bar{x})$, then $\varphi_{o}$ is Lipschitz continuous around $\bar{x}$.

Proof. The proof follows along the lines of the proof of Theorem 5.2 while applying Theorem 3.6 to the representation of the two-level value function in Lemma 5.1.

To consider $S$-type upper bounds for the limiting subdifferential of $\varphi_{o}$, define the set $\Lambda_{y}^{e s}(\bar{x}, \bar{y}, \bar{u})$ similarly to $\Lambda_{y}^{e m}(\bar{x}, \bar{y}, \bar{u})$ by replacing $(4.7)$ by

$$
\forall i \in \theta, \beta_{i} \geq 0 \wedge \sum_{l=1}^{m} \gamma_{l} \nabla_{y_{l}} g_{i}(\bar{x}, \bar{y}) \geq 0
$$

and arriving at the next sensitivity result under the modified $S$-qualification conditions

$$
\begin{aligned}
& \left.\begin{array}{rl}
\nabla g(\bar{x}, \bar{y})^{\top} \beta+\nabla_{x, y} \mathcal{L}(\bar{x}, \bar{y}, \bar{u})^{\top} \gamma=0 \\
\nabla_{y} g_{\nu}(\bar{x}, \bar{y}) \gamma=0, \beta_{\eta}=0
\end{array}\right\} \Longrightarrow\left\{\begin{array}{l}
\beta_{\theta}=0, \\
\nabla_{y} g_{\theta}(\bar{x}, \bar{y}) \gamma=0,
\end{array}\right. \\
& \left.\begin{array}{r}
\nabla_{y} g(\bar{x}, \bar{y})^{\top} \beta+\nabla_{y} \mathcal{L}(\bar{x}, \bar{y}, \bar{u})^{\top} \gamma=0 \\
\nabla_{y} g_{\nu}(\bar{x}, \bar{y}) \gamma=0, \beta_{\eta}=0
\end{array}\right\} \Longrightarrow \beta=0, \gamma=0 .
\end{aligned}
$$

Copyright (C) by SIAM. Unauthorized reproduction of this article is prohibited. 
Theorem 5.4 (S-type sensitivity analysis for the two-level value function). Let the lower-level problem (1.2) be convex, and let the lower-level regularity (4.2) be satisfied at $(\bar{x}, y)$ for all $y \in S(\bar{x})$. The following assertions hold:

(i) Assume that the optimal solution map $S_{o}^{h}(5.5)$ is inner semicontinuous at $(\bar{x}, \bar{y}, \bar{u})$, where conditions $\left(A_{1}^{m}\right)$ and $\left(A_{1}^{s}\right)$ are satisfied. Then we have

$$
\partial \varphi_{o}(\bar{x}) \subset \bigcup_{(\beta, \gamma) \in \Lambda_{y}^{e s}(\bar{x}, \bar{y}, \bar{u})}\left\{\nabla_{x} F(\bar{x}, \bar{y})+\nabla_{x} g(\bar{x}, \bar{y})^{\top} \beta+\nabla_{x} \mathcal{L}(\bar{x}, \bar{y}, \bar{u})^{\top} \gamma\right\} .
$$

Furthermore, if conditions $\left(A_{1}^{m}\right)$ and $\left(A_{1}^{s}\right)$ are replaced with $\left(A_{2}^{s}\right)$, then $\varphi_{o}$ is strictly differentiable at $\bar{x}$, and its gradient is calculated by

$$
\nabla \varphi_{o}(\bar{x})=\nabla_{x} F(\bar{x}, \bar{y})+\nabla_{x} g(\bar{x}, \bar{y})^{\top} \beta+\nabla_{x} \mathcal{L}(\bar{x}, \bar{y}, \bar{u})^{\top} \gamma,
$$

where $\{(\beta, \gamma)\}=\Lambda_{y}^{e s}(\bar{x}, \bar{y}, \bar{u})$ is the unique multiplier.

(ii) Let $S_{o}^{h}(5.5)$ be inner semicompact at $\bar{x}$, and let conditions $\left(A_{1}^{m}\right)$ and $\left(A_{1}^{s}\right)$ hold at $(\bar{x}, \bar{y}, \bar{u})$ for all $(\bar{y}, \bar{u}) \in S_{o}^{h}(\bar{x})$. Then we have

$$
\partial \varphi_{o}(\bar{x}) \subset \bigcup_{(\bar{y}, \bar{u}) \in S_{o}^{h}(\bar{x})} \bigcup_{(\beta, \gamma) \in \Lambda_{y}^{e s}(\bar{x}, \bar{y}, \bar{u})}\left\{\nabla_{x} F(\bar{x}, \bar{y})+\nabla_{x} g(\bar{x}, \bar{y})^{\top} \beta+\nabla_{x} \mathcal{L}(\bar{x}, \bar{y}, \bar{u})^{\top} \gamma\right\} .
$$

Proof. Considering the representation of the two-level value function $\varphi_{o}$ in (5.3) (cf. also (5.7)), it is obvious that $\left(A_{1}^{s}\right)$ corresponds to the qualification condition $\left(A_{1}^{3}\right)$ in (3.18). Thus the inclusion in (i) of this theorem follows from that of Theorem 3.7(i) by taking into account the estimate of $\partial \varphi_{o}$ in Theorem 5.2(i). Furthermore, the strict differentiability of $\varphi_{o}$ and the expression of its gradient follow from Corollary 3.8 by noting that assumption $\left(A_{2}^{s}\right)$ is the counterpart of $\left(A_{2}^{3}\right)$ from (3.19) for the OPCC reformulation of $\varphi_{o}$ in (5.3). The estimate of the limiting subdifferential of $\varphi_{o}$ in Theorem 5.4(ii) is derived similarly by using Theorem 3.7(ii).

Denoting by $B_{A}(\bar{x}, \bar{y}, \bar{u})$ for $A:=M, C, S$, the upper bound of $\partial \varphi_{o}(\bar{x})$ in Theorems 5.2(i), 5.3(i), and 5.4(i), respectively, we observe that

$$
B_{S}(\bar{x}, \bar{y}, \bar{u}) \subset B_{M}(\bar{x}, \bar{y}, \bar{u}) \subset B_{C}(\bar{x}, \bar{y}, \bar{u}) .
$$

Indeed, it follows from $\Lambda_{y}^{e s}(\bar{x}, \bar{y}, \bar{u}) \subset \Lambda_{y}^{e m}(\bar{x}, \bar{y}, \bar{u}) \subset \Lambda_{y}^{e c}(\bar{x}, \bar{y}, \bar{u})$. A similar observation can be made for the upper bounds obtained under inner semicompactness.

Now in the $M$-case we provide different types of upper bounds of the two-level value function via the OPEC approach from section 4. Although the M-type sensitivity analysis of $\varphi_{o}$ would be self-evident by means of Theorem 4.1 while considering the OPEC representation of $\varphi_{o}$, we nevertheless state it here in order to have a clear picture of the differences with the sensitivity result of Theorem 5.2. To proceed, denote $h(x, y):=\nabla_{y} f(x, y)$ in the setting of section 4 and also $\left(A_{1}^{e}\right):=\left(A_{1}^{4}\right),\left(A_{2}^{e}\right):=\left(A_{2}^{4}\right)$, $\left(A_{3}^{e}\right):=\left(A_{3}^{4}\right)$, and $\left(A_{4}^{e}\right):=\left(A_{4}^{4}\right)$. Define further the two-level solution map by

$$
S_{o}(x):=\left\{y \mid 0 \in \nabla_{y} f(x, y)+N_{K(x)}(y), F(x, y) \leq \varphi_{o}(x)\right\} .
$$

Then we get the following sensitivity results via the OPEC approach.

Corollary 5.5 (M-type sensitivity analysis via the OPEC reformulation). Providing that the lower-level problem (1.2) is convex, we have the following:

(i) Assume that the optimal solution map $S_{o}$ in (5.14) is inner semicontinuous at $(\bar{x}, \bar{y})$, where the lower-level regularity (4.2) and conditions $\left(A_{1}^{e}\right)$ and $\left(A_{2}^{e}\right)$ hold. Then

$$
\partial \varphi_{o}(\bar{x}) \subset \bigcup_{\bar{u} \in \Lambda(\bar{x}, \bar{y})} \bigcup_{(\beta, \gamma) \in \Lambda_{y}^{e m}(\bar{x}, \bar{y}, \bar{u})}\left\{\nabla_{x} F(\bar{x}, \bar{y})+\nabla_{x} g(\bar{x}, \bar{y})^{\top} \beta+\nabla_{x} \mathcal{L}(\bar{x}, \bar{y}, \bar{u})^{\top} \gamma\right\} .
$$

Copyright $@$ by SIAM. Unauthorized reproduction of this article is prohibited. 
If in addition $\left(A_{3}^{e}\right)$ is satisfied at $(\bar{x}, \bar{y})$, then $\varphi_{o}$ is Lipschitz continuous around $\bar{x}$.

(ii) Let $S_{o}$ be inner semicompact at $\bar{x}$, and let $\left(A_{1}^{e}\right),\left(A_{2}^{e}\right)$ and the lower-level regularity $(4.2)$ be satisfied at $(\bar{x}, \bar{y})$ for all $\bar{y} \in S_{o}(\bar{x})$. Then we have

$$
\begin{aligned}
\partial \varphi_{o}(\bar{x}) \subset \bigcup_{\bar{y} \in S_{o}(\bar{x})} \bigcup_{\bar{u} \in \Lambda(\bar{x}, \bar{y})} \bigcup_{(\beta, \gamma) \in \Lambda_{y}^{e m}(\bar{x}, \bar{y}, \bar{u})}\left\{\nabla_{x} F(\bar{x}, \bar{y})+\right. & \nabla_{x} g(\bar{x}, \bar{y})^{\top} \beta \\
& \left.+\nabla_{x} \mathcal{L}(\bar{x}, \bar{y}, \bar{u})^{\top} \gamma\right\} .
\end{aligned}
$$

If $\left(A_{3}^{e}\right)$ also holds at $(\bar{x}, \bar{y})$ for $\bar{y} \in S_{o}(\bar{x})$, then $\varphi_{o}$ is locally Lipschitzian around $\bar{x}$.

Proof. The convexity of the lower-level problem (1.2) ensures the following OPEC representation of the two-level value function $\varphi_{o}$ :

$$
\varphi_{o}(x)=\min _{y}\left\{F(x, y) \mid 0 \in \nabla_{y} f(x, y)+N_{K(x)}(y)\right\},
$$

derived from the generalized equation reformulation of the solution map (1.5):

$$
S(x)=\left\{y \mid 0 \in \nabla_{y} f(x, y)+N_{K(x)}(y)\right\} .
$$

Then assertions (i) and (ii) from Theorem 4.1 applied to (5.15) give the result.

Remark 5.6 (on the differences between the M-type sensitivity analysis of the two-level value function via the OPCC and OPEC reformulations). The difference between the upper estimate of $\partial \varphi_{o}$ in Theorem 5.2(i) and that in Corollary 5.5(i) is clear: the latter contains the union over $\Lambda(\bar{x}, \bar{y})$, which makes it larger than that obtained in the former result. The estimates in Theorem 5.2(ii) and Corollary 5.5(ii) seem to be closer in the sense that $\bar{y} \in S_{o}(\bar{x})$ if and only if there exists $\bar{u}$ such that $(\bar{y}, \bar{u}) \in S_{o}^{h}(\bar{x}):=\left\{(y, u) \mid u \in \Lambda(\bar{x}, y), F(\bar{x}, y) \leq \varphi_{o}(\bar{x})\right\}$. On the qualification conditions we observe that, although similar, they are not identical. However, the strongest ones, $\left(A_{3}^{m}\right)$ and $\left(A_{4}^{e}\right)$, are the same, but as already noted the results are not the same for the subdifferential estimates of the two-level value function $\varphi_{o}$. This observation is similar to that made in [14] on relationships between the stationarity conditions obtained via the KKT and primal KKT/OPEC approaches. The principal difference between the results of this type is that the appearance of the lower-level multipliers $\bar{u}$ in Corollary 5.5 is a posteriori while it is a priori in Theorem 5.2.

To conclude this subsection, some comments on the qualifications conditions used here are in order. Following Remark 3.3, we conclude that condition $\left(A_{1}^{m}\right)$ can be replaced by the weaker calmness property of the set-valued mapping

$$
\Psi(z, \vartheta):=\left\{(x, y, u) \mid \mathcal{L}(x, y, u)+z=0,\left(-g_{i}(x, y), u_{i}\right)+\vartheta_{i} \in \Lambda_{i}, i:=1, \ldots, p\right\}
$$

where $\Lambda_{i}:=\left\{(a, b) \in \mathbb{R}^{2} \mid a \geq 0, b \geq 0, a b=0\right\}$. The latter assumption is automatic when the mappings $g$ and $(x, y) \mapsto \nabla_{y} f(x, y)$ are linear. Condition $\left(A_{3}^{m}\right)$ can replace the two other assumptions $\left(A_{1}^{m}\right)$ and $\left(A_{2}^{m}\right)$. Note, however, that in the case of the LLVF reformulation considered in the next subsection, the latter sequence of implication between the constraint qualifications will not be available. Also observe that comments similar to the above can be stated for the C-qualification conditions in (5.12).

5.2. LLVF approach. In this subsection we develop the lower-level value function (LLVF) approach to sensitivity analysis of the two-level value function $\varphi_{o}$ from (5.1). Let us start by recalling that the argminimum/solution map of the lower-level problem (1.2) can be written as

$$
S(x):=\{y \mid f(x, y)-\varphi(x) \leq 0, g(x, y) \leq 0\},
$$


with $\varphi$ denoting the value function associated to the lower-level problem (1.2), i.e.,

$$
\varphi(x):=\min _{y}\{f(x, y) \mid g(x, y) \leq 0\} .
$$

Hence we have the LLVF reformulation of the two-level value function $\varphi_{o}$ written as

$$
\varphi_{o}(x):=\min _{y}\{F(x, y) \mid g(x, y) \leq 0, f(x, y)-\varphi(x) \leq 0\} .
$$

Since our basic subdifferential $\partial \varphi$ does not satisfy the plus/minus symmetry, an appropriate estimate of $\partial(-\varphi)$ is needed to proceed with this approach. It can be done by invoking the well-known convex hull property

$$
\operatorname{co} \partial(-\varphi)(\bar{x})=-\operatorname{co} \partial \varphi(\bar{x})
$$

for local Lipschitzian functions, which follows from [5] due to $\bar{\partial} \varphi(\bar{x})=\operatorname{co} \partial \varphi(\bar{x})$. The next theorem collects the results in this direction needed in what follows.

THEOREM 5.7 (sensitivity analysis of the negative value function in the lowerlevel problem). The following assertions hold for the value function $\varphi$ in (5.18):

(i) If the solution map $S$ in (1.5) is inner semicompact at $\bar{x}$, and $(\bar{x}, y)$ is lowerlevel regular (4.2) for all $y \in S(\bar{x})$, then $\varphi$ is Lipschitz continuous around $\bar{x}$ with

$$
\begin{aligned}
& \partial(-\varphi)(\bar{x}) \subset\left\{-\sum_{s=1}^{n+1} v_{s}\left(\nabla_{x} f\left(\bar{x}, y_{s}\right)+\nabla_{x} g\left(\bar{x}, y_{s}\right)^{\top} \mu_{s}\right) \mid \sum_{s=1}^{n+1} v_{s}=1,\right. \\
&\left.\forall s=1, \ldots, n+1, \quad v_{s} \geq 0, \quad y_{s} \in S(\bar{x}), \quad \mu_{s} \in \Lambda\left(\bar{x}, y_{s}\right)\right\} .
\end{aligned}
$$

(ii) Assume that $(\bar{x}, \bar{y})$ is lower-level regular (4.2), and that either $S$ (1.5) is inner semicontinuous at $(\bar{x}, \bar{y})$ or $f$ and $g_{i}, i=1, \ldots, p$, are fully convex. Then $\varphi$ is Lipschitz continuous around $\bar{x}$ and the basic subdifferential of $-\varphi$ is estimated as

$$
\partial(-\varphi)(\bar{x}) \subset \bigcup_{\mu \in \Lambda(\bar{x}, \bar{y})}\left\{-\nabla_{x} f(\bar{x}, \bar{y})-\sum_{i=1}^{p} \mu_{i} \nabla_{x} g_{i}(\bar{x}, \bar{y})\right\} .
$$

Proof. The local Lipschitz continuity of $\varphi$ is justified in [40] under the fulfillment of the lower-level regularity (4.2) in both inner semicontinuous and inner semicompactness cases. If the functions $f$ and $g_{i}$ as $i=1, \ldots, p$ are fully convex, then the value function $\varphi$ is convex as well; in this case the Lipschitz continuity follows from [5]. To prove the subdifferential inclusion in (i), recall by [43] that

$$
\partial \varphi(\bar{x}) \subset \bigcup_{y \in S(\bar{x})} \bigcup_{\mu \in \Lambda(\bar{x}, y)}\left\{\nabla_{x} f(\bar{x}, y)+\sum_{i=1}^{p} \mu_{i} \nabla_{x} g_{i}(\bar{x}, \bar{y})\right\}
$$

under the assumptions made in (i). The claimed estimate of $\partial(-\varphi)$ follows from here by the classical Carathéodory theorem while noting that

$$
\partial(-\varphi)(\bar{x}) \subset \operatorname{co} \partial(-\varphi)(\bar{x})=-\operatorname{co} \partial \varphi(\bar{x})
$$

where the second equality follows from the convex hull property (5.20).

When $S$ is inner semicontinuous at $(\bar{x}, \bar{y})$, we have from [41] that

$$
\bar{\partial} \varphi(\bar{x}) \subset \bigcup_{\mu \in \Lambda(\bar{x}, \bar{y})}\left\{\nabla_{x} f(\bar{x}, \bar{y})+\sum_{i=1}^{p} \mu_{i} \nabla_{x} g_{i}(\bar{x}, \bar{y})\right\},
$$

Copyright $@$ by SIAM. Unauthorized reproduction of this article is prohibited. 
which implies (5.21) similarly to the previous case. Estimate (5.21) in the convex case follows from (5.23) and the fact that $\bar{\partial} \varphi(\bar{x})=\partial \varphi(\bar{x})$ in this case.

Note that in the fully convex (even nonsmooth) case the lower-level regularity assumption in Theorem 5.7 can be replaced by a much weaker qualification condition [16] requiring that the set

$$
\text { epi } f^{*}+\text { cone }\left(\bigcup_{i=1}^{p} \text { epi } g_{i}^{*}\right) \text { is closed on } \mathbb{R}^{n} \times \mathbb{R}^{m} \times \mathbb{R},
$$

where epi $\vartheta^{*}$ denotes the conjugate function for an extended-real-valued convex function $\vartheta$. Note also that inclusion (5.7) can similarly be deduced from the well-known (see, e.g., [19, 23]) upper estimate of the generalized gradient of value functions.

Next we observe that calculating the coderivative of the optimal solution map $S$ from (5.17) is highly significant in our approach. In the current situation this reduces to computing the limiting normal cone to the graph of $S$ :

$$
\operatorname{gph} S=\{(x, y) \in \Omega \mid f(x, y)-\varphi(x) \leq 0\} \text { with } \Omega:=\{(x, y) \mid g(x, y) \leq 0\}
$$

in terms of the initial data. To proceed in this way using the conventional results of the generalized differential calculus [38, 39] requires the fulfillment of the basic qualification condition, which reads in this case as

$$
\partial(f-\varphi)(\bar{x}, \bar{y}) \cap\left(-N_{\Omega}(\bar{x}, \bar{y})\right)=\emptyset .
$$

However, it is shown in [13] that condition (5.26) fails in common situations, in particular, when $\varphi$ is locally Lipschitzian around the point in question. The following weaker assumption helps circumvent this difficulty:

$\left(A_{1}^{v}\right) \quad$ the mapping $\Xi(v):=\{(x, y) \in \Omega \mid f(x, y)-\varphi(x) \leq v\}$ is calm at $(0, \bar{x}, \bar{y})$.

Applying the concept of stability regions known in linear programming (see, e.g., [8]) to the optimal value function $\varphi$ makes it possible to show, by means of Robinson's theorem [49] on the upper-Lipschitz continuity of a polyhedral set-valued mapping, that $\left(A_{1}^{v}\right)$ is automatic if $f$ and $g$ are linear. Furthermore, condition $\left(A_{1}^{v}\right)$ is satisfied at $(\bar{x}, \bar{y})$ for locally Lipschitzian functions $\varphi$ if we pass to the boundary of the normal cone in (5.26), i.e., if the following qualification condition holds:

$$
\partial(f-\varphi)(\bar{x}, \bar{y}) \cap\left(-\operatorname{bd} N_{\Omega}(\bar{x}, \bar{y})\right)=\emptyset
$$

with $\Omega$ being semismooth (in particular, convex); cf. [13, 25]. Condition (5.27) seems to be especially effective for the so-called simple convex bilevel optimization problems $[9,13]$. It is worth mentioning that for the latter class, condition (5.27) can be further weakened [13] by passing to the boundary of the subdifferential of $f$.

Another sufficient condition for the validity of $\left(A_{1}^{v}\right)$ is provided by the notion of uniform weak sharp minima. Recall that problem (1.2) is said to have a uniform weak sharp minimum around $(\bar{x}, \bar{y})$ if there exist positive numbers $\lambda$ and $\delta$ such that

$$
f(x, y)-\varphi(x) \geq \lambda d(y, S(x)) \text { for all }(x, y) \in B((\bar{x}, \bar{y}), \delta) \cap \Omega .
$$

The concept of uniform weak sharp minimum, which emerged from the notions of sharp minimum introduced by Polyak [48] and weak sharp minimum introduced by Ferris [18], was developed by Ye and Zhu [61], while the above localized version (5.28) 
has been recently considered by Henrion and Surowiec [27] and by Mordukhovich, Nam, and Phan [41]. It follows from [27, Proposition 3.8] that $\left(A_{1}^{v}\right)$ holds at $(\bar{x}, \bar{y})$ if problem (1.2) has a uniform weak sharp minimum around $(\bar{x}, \bar{y})$. Furthermore, it is shown in [41] that if $f$ and $g$ are linear in $y$ and $(x, y)$, respectively, then the lowerlevel problem has a local uniform weak sharp minimum. A number of other efficient conditions ensuring a uniform weak sharp minimum in bilevel programming can be found in $[11,13,16,41,56,61]$. Finally note that notion (5.28) is closely related to the partial calmness property introduced in [61]; condition $\left(A_{1}^{v}\right)$ is in fact sufficient for the partial calmness of the bilevel program in question if we drop the upper-level constraint or include it in the constraint set $\Omega$.

Considering further the Lipschitz-like property of the solution map $S$ in (5.17), we impose the following additional qualification condition:

$\left(A_{2}^{v}\right) \quad\left[(\lambda, \beta) \in \Lambda_{y}^{o}(\bar{x}, \bar{y}, 0), u \in \partial(-\varphi)(\bar{x})\right] \Longrightarrow \lambda u=-\lambda \nabla_{x} f(\bar{x}, \bar{y})-\nabla_{x} g(\bar{x}, \bar{y})^{\top} \beta$,

where $\Lambda_{y}^{o}(\bar{x}, \bar{y}, v)$ for $v \in \mathbb{R}^{m}$ denotes a particular set of multipliers that plays a significant role in the rest of the section:

$$
\begin{aligned}
\Lambda_{y}^{o}(\bar{x}, \bar{y}, v):=\{(\lambda, \beta) \mid v & +\lambda \nabla_{y} f(\bar{x}, \bar{y})+\sum_{i=1}^{p} \beta_{i} \nabla_{y} g_{i}(\bar{x}, \bar{y})=0, \\
\lambda & \left.\geq 0, \beta_{i} \geq 0, \beta_{i} g_{i}(\bar{x}, \bar{y})=0, i=1, \ldots, p\right\} .
\end{aligned}
$$

The next proposition describes an important setting where assumption $\left(A_{2}^{v}\right)$ holds.

Proposition 5.8 (validity of assumption $\left(A_{2}^{v}\right)$ ). Let $f: \mathbb{R}^{n} \times \mathbb{R}^{m} \rightarrow \mathbb{R}$ and $g_{i}: \mathbb{R}^{m} \rightarrow \mathbb{R}, i=1, \ldots, p$, be convex and smooth. Consider the value function $\varphi(x):=\min _{y}\left\{f(x, y) \mid g_{i}(y) \leq 0, i=1, \ldots, p\right\}$ and the corresponding solution map $S(x)=\min _{y}\left\{f(x, y) \mid g_{i}(y) \leq 0, i=1, \ldots, p\right\}$. Take $(\bar{x}, \bar{y}) \in \operatorname{gph} S$ with $\varphi(\bar{x})<\infty$ and assume that there exists $\hat{y}$ with $g_{i}(\hat{y})<0$ for $i=1, \ldots, p$. Then $\left(A_{2}^{v}\right)$ holds at $(\bar{x}, \bar{y})$.

Proof. Under the setting of this proposition, it follows from the convex case of Theorem 5.7(ii) that the function $-\varphi$ is strictly differentiable at $\bar{x}$ and $\partial(-\varphi)(\bar{x})=$ $\left\{-\nabla_{x} f(\bar{x}, \bar{y})\right\}$, which therefore justifies our conclusion.

In the next theorem we use the above qualification conditions to derive a coderivative estimate and Lipschitz-like property for lower-level solution maps via the LLVF reformulation in (5.17).

TheOREM 5.9 (coderivative estimate and Lipschitz-like property of lower-level solution maps). Let mapping $S$ (5.17) be inner semicontinuous at $(\bar{x}, \bar{y}) \in \operatorname{gph} S$, and let the condition $\left(A_{1}^{v}\right)$ and the lower-level regularity (4.2) be satisfied at this point. Then for all $v \in \mathbb{R}^{m}$ we have the coderivative estimate

$$
D^{*} S(\bar{x}, \bar{y})(v) \subset \bigcup_{(\lambda, \beta) \in \Lambda_{y}^{o}(\bar{x}, \bar{y}, v)}\left\{\lambda\left(\nabla_{x} f(\bar{x}, \bar{y})+\partial(-\varphi)(\bar{x})\right)+\nabla_{x} g(\bar{x}, \bar{y})^{\top} \beta\right\} .
$$

If in addition $\left(A_{2}^{v}\right)$ holds at $(\bar{x}, \bar{y})$, then $S$ is Lipschitz-like around this point.

Proof. It follows from Theorem 5.7(ii) that the lower-level value function $\varphi$ is Lipschitz continuous around $\bar{x}$ under the lower-level regularity and the inner semicontinuity assumption. If we add the calmness property $\left(A_{1}^{v}\right)$, then

$$
N_{\operatorname{gph} S}(\bar{x}, \bar{y}) \subset \bigcup_{\lambda \geq 0}\left\{\lambda(\nabla f(\bar{x}, \bar{y})+\partial(-\varphi)(\bar{x}) \times\{0\})+N_{\Omega}(\bar{x}, \bar{y})\right\}
$$

by $[24$, Theorem 4.1] while taking into account that the constraint $f(x, y)-\varphi(x) \leq 0$ is active at $(\bar{x}, \bar{y})$. The coderivative estimate in the theorem follows now from definition (2.6) and the well-known expression of the normal cone 


$$
N_{\Omega}(\bar{x}, \bar{y})=\left\{\nabla g(\bar{x}, \bar{y})^{\top} \beta \mid \beta \geq 0, \beta^{\top} g(\bar{x}, \bar{y})=0\right\},
$$

which holds under the the lower-level regularity at $(\bar{x}, \bar{y})$. Further, by the inclusion in the theorem, and the coderivative criterion (2.9) for the Lipschitz-like property, we get that it holds if

$$
\left.\begin{array}{r}
u \in \lambda\left(\nabla_{x} f(\bar{x}, \bar{y})+\partial(-\varphi)(\bar{x})\right)+\nabla_{x} g(\bar{x}, \bar{y})^{\top} \beta \\
(\lambda, \beta) \in \Lambda_{y}^{o}(\bar{x}, \bar{y}, 0)
\end{array}\right\} \Longrightarrow u=0
$$

which is in fact equivalent to the assumed qualification condition $\left(A_{2}^{v}\right)$.

Remark 5.10 (sensitivity result of the solution map $S$ under full convexity and inner semicompactness). It follows from the alternative statement in Theorem 5.7(ii) that the inner semicontinuity of $S$ can be dropped in the assumptions of Theorem 5.9 if the functions $f$ and $g$ are fully convex. As usual, the inner semicontinuity can be replaced by inner semicompactness with a larger inclusion in Theorem 5.9.

To conduct a local sensitivity analysis of the two-level value function $\varphi_{o}$ defined in (5.19), we associate with it the optimal solution map $S_{o}$, which reads as follows:

$$
S_{o}(x)=\left\{y \in K(x) \mid f(x, y) \leq \varphi(x), F(x, y) \leq \varphi_{o}(x)\right\} .
$$

Having in mind the definition of the multiplier set $\Lambda_{y}^{o}(\bar{x}, \bar{y}, v)$ in (5.29), we put $\Lambda_{y}^{o}(\bar{x}, \bar{y}):=\Lambda_{y}^{o}\left(\bar{x}, \bar{y}, \nabla_{y} F(\bar{x}, \bar{y})\right)$. Then sensitivity results for $\varphi_{o}$ are given next.

THEOREM 5.11 (LLVF approach to sensitivity analysis for the two-level value function). Considering the LLVF reformulation (5.19) of the two-level value function, the following assertions hold:

(i) Assume that $S_{o}$ in (5.30) is inner semicontinuous at $(\bar{x}, \bar{y})$ and that condition $\left(A_{1}^{v}\right)$ and the lower-level regularity (4.2) are satisfied at this point. Then we have

$$
\partial \varphi_{o}(\bar{x}) \subset \bigcup_{(\lambda, \beta) \in \Lambda_{y}^{o}(\bar{x}, \bar{y})} \bigcup_{\mu \in \Lambda(\bar{x}, \bar{y})}\left\{\nabla_{x} F(\bar{x}, \bar{y})+\sum_{i=1}^{p}\left(\beta_{i}-\lambda \mu_{i}\right) \nabla_{x} g_{i}(\bar{x}, \bar{y})\right\} .
$$

If in addition $\left(A_{2}^{v}\right)$ holds at $(\bar{x}, \bar{y})$, then $\varphi_{o}$ is Lipschitz continuous around $\bar{x}$.

(ii) Assume that $S_{o}$ is inner semicompact at $\bar{x}$, that the lower-level regularity (4.2) holds at $(\bar{x}, y)$ for all $y \in S(\bar{x})$, and that $\left(A_{1}^{v}\right)$ holds at $(\bar{x}, y)$ for all $y \in S_{o}(\bar{x})$. Then

$$
\begin{aligned}
& \partial \varphi_{o}(\bar{x}) \subset \bigcup_{y \in S_{o}(\bar{x})} \bigcup_{(\lambda, \beta) \in \Lambda_{y}^{o}(\bar{x}, y)}\left\{\nabla_{x} F(\bar{x}, y)+\lambda \nabla_{x} f(\bar{x}, y)\right. \\
& \left.+\lambda \partial(-\varphi)(\bar{x})+\nabla_{x} g(\bar{x}, y)^{\top} \beta\right\},
\end{aligned}
$$

where the subdifferential $\partial(-\varphi)(\bar{x})$ is estimated in Theorem $5.7(\mathrm{i})$. If in addition $\left(A_{2}^{v}\right)$ holds at $(\bar{x}, y)$ for all $y \in S_{o}(\bar{x})$, then $\varphi_{o}$ is Lipschitz continuous around $\bar{x}$.

Proof. To justify (i), observe by Theorem 2.1(i) that

$$
\partial \varphi_{o}(\bar{x}) \subset \nabla_{x} F(\bar{x}, \bar{y})+D^{*} S(\bar{x}, \bar{y})\left(\nabla_{y} F(\bar{x}, \bar{y})\right)
$$

under the inner semicontinuity assumption on $S_{o}$. Since we have $S_{o}(x) \subset S(x)$ for all $x \in X$, the lower-level optimal solution map $S$ in (5.17) is also inner semicontinuous at $(\bar{x}, \bar{y}) \in \operatorname{gph} S_{o}$. Thus the upper estimate of $\partial \varphi_{o}(\bar{x})$ in this theorem follows from those for the coderivative of $S$ in Theorem 5.9 and for the subdifferential of the lower-level value function $\varphi$ in Theorem 5.7(ii). To justify the local Lipschitz continuity of $\varphi_{o}$ in 
(i) under $\left(A_{2}^{v}\right)$, recall that the latter condition implies the Lipschitz-like property of $S$ around $(\bar{x}, \bar{y})$ by Theorem 5.9. Thus we have the claimed result from Theorem 2.1(i). Assertion (ii) is proved similarly following the discussion in Remark 5.10.

Observe that the upper bound of the subdifferential estimate in Theorem 5.11(i) does not depend on the partial derivative of the lower-level cost function $f$ with respect to the upper-level variable $x$. In the next section this will induce in the context of necessary optimality conditions for the original optimistic formulation $\left(\mathrm{P}_{o}\right)$ a remarkable phenomenon first discovered in [11] in the framework of the auxiliary problem (P). Note that such a phenomenon is no longer true if the inner semicontinuity assumption on $S_{o}$ is replaced by the inner semicompactness one in assertion (ii) of Theorem 5.11. Let us also mention that the inner semicompactness of $S_{o}$ in Theorem 5.11(ii) can be replaced by the easier yet some what more restrictive uniform boundedness assumption imposed on $S_{o}$ or even on the lower-level solution map $S$.

Remark 5.12 (convexity in the two-level value function). In the case of the standard value function $\varphi$ in (5.18), its convexity plays an important role in the sensitivity result of Theorem 5.7. Note, however, that such an idea is not applicable to the twolevel value function $\varphi_{o}$ in (5.1) for the simple reason that the graph of the lower-level solution map $S(5.17)$ is usually nonconvex. Therefore, it does not make sense to consider the convexity of $\varphi_{o}$ as an alternative to the inner semicontinuity in Theorem 5.11(i). This is in accordance with the well-known fact that inner semicontinuity and convexity are not related; cf., e.g., [11]. Nonetheless, if instead the lower-level value function $\varphi$ (5.18) is convex (which is quite possible), the upper estimate of $\partial(-\varphi)(\bar{x})$ in Theorem 5.7(ii) can well be inserted in the upper bound of $\partial \varphi_{o}(\bar{x})$ in Theorem 5.11(ii) in order to have a simpler expression.

Denote by $B_{V}(\bar{x}, \bar{y})$ the upper bound of the basic subdifferential of $\varphi_{o}$ in Theorem 5.11(i). Recall (cf. the discussion following Theorem 5.4) that $B_{S}(\bar{x}, \bar{y}, \bar{u}) \subset$ $B_{M}(\bar{x}, \bar{y}, \bar{u}) \subset B_{C}(\bar{x}, \bar{y}, \bar{u})$ in the framework of upper bounds for $\partial \varphi_{o}$ obtained via the OPCC approach. Now note that there is a significant difference between the latter classes of bounds and those obtained via the LLVF approach (cf. Theorem 5.11), namely, due to the presence of the second order term $\nabla_{x, y} \mathcal{L}(\bar{x}, \bar{y}, \bar{u})^{\top} \gamma$ in the OPCC case. It is shown in the next result that if this term is zero, then we have the inclusion $B_{S}(\bar{x}, \bar{y}, \bar{u}) \subset B_{V}(\bar{x}, \bar{y})$ if $\Lambda_{y}^{o}(\bar{x}, \bar{y})$ from (5.29) is defined with $\lambda$ free, i.e., $\lambda \in \mathbb{R}$.

Proposition 5.13 (comparing subdifferential estimates of the two-level value function). Let $b(\bar{x}, \bar{y}, \bar{u}, \beta, \gamma) \in B_{S}(\bar{x}, \bar{y}, \bar{u})$ for some $(\beta, \gamma)$ with $\nabla_{x, y} \mathcal{L}(\bar{x}, \bar{y}, \bar{u})^{\top} \gamma=0$. Then we have $b(\bar{x}, \bar{y}, \bar{u}, \beta, \gamma) \in B_{V}(\bar{x}, \bar{y})$, provided that $\lambda$ is free in $\Lambda_{y}^{o}(\bar{x}, \bar{y})$.

Proof. If $b(\bar{x}, \bar{y}, \bar{u}, \beta, \gamma) \in B_{S}(\bar{x}, \bar{y}, \bar{u})$ for some $(\beta, \gamma)$, then conditions (4.6) and (5.13) together with the following ones are satisfied:

$$
\begin{aligned}
&-b(\bar{x}, \bar{y}, \bar{u}, \beta, \gamma)+ \nabla_{x} F(\bar{x}, \bar{y})+\nabla_{x} g(\bar{x}, \bar{y})^{\top} \beta+\nabla_{x} \mathcal{L}(\bar{x}, \bar{y}, \bar{u})^{\top} \gamma=0, \\
& \nabla_{y} F(\bar{x}, \bar{y})+\nabla_{y} g(\bar{x}, \bar{y})^{\top} \beta+\nabla_{y} \mathcal{L}(\bar{x}, \bar{y}, \bar{u})^{\top} \gamma=0 .
\end{aligned}
$$

It follows from these conditions by [64, proof of Theorem 3.9] that there exist a vector $\beta^{*}$ and a real number $\lambda^{*}$ such that we have

$$
\begin{array}{r}
-b(\bar{x}, \bar{y}, \bar{u}, \beta, \gamma)+\nabla_{x} F(\bar{x}, \bar{y})+\nabla_{x} g(\bar{x}, \bar{y})^{\top}\left(\beta^{*}-\lambda^{*} \bar{u}\right) \\
+\nabla_{x} \mathcal{L}(\bar{x}, \bar{y}, \bar{u})^{\top} \gamma=0, \\
\nabla_{y} F(\bar{x}, \bar{y})+\nabla_{y} g(\bar{x}, \bar{y})^{\top}\left(\beta^{*}-\lambda^{*} \bar{u}\right)+\nabla_{y} \mathcal{L}(\bar{x}, \bar{y}, \bar{u})^{\top} \gamma=0 \\
\text { for } i=1, \ldots, p, \beta_{i}^{*} \geq 0, \beta_{i}^{*} g_{i}(\bar{x}, \bar{y})=0,
\end{array}
$$

with $\bar{u} \in \Lambda(\bar{x}, \bar{y})$. If $\nabla_{x, y} \mathcal{L}(\bar{x}, \bar{y}, \bar{u})^{\top} \gamma=0$, then (5.31) and (5.32) reduce to 


$$
\begin{array}{r}
b(\bar{x}, \bar{y}, \bar{u}, \beta, \gamma)=\nabla_{x} F(\bar{x}, \bar{y})+\nabla_{x} g(\bar{x}, \bar{y})^{\top}\left(\beta^{*}-\lambda^{*} \bar{u}\right) \\
\text { and } \nabla_{y} F(\bar{x}, \bar{y})+\nabla_{y} g(\bar{x}, \bar{y})^{\top}\left(\beta^{*}-\lambda^{*} \bar{u}\right)=0,
\end{array}
$$

respectively. Now observe that $-\nabla_{y} g(\bar{x}, \bar{y})^{\top} \bar{u}=\nabla_{y} f(\bar{x}, \bar{y})$ with $\bar{u} \in \Lambda(\bar{x}, \bar{y})$. Inserting this into (5.35) gives us the equation

$$
\nabla_{y} F(\bar{x}, \bar{y})+\lambda^{*} \nabla_{y} f(\bar{x}, \bar{y})+\nabla_{y} g(\bar{x}, \bar{y})^{\top} \beta^{*}=0 .
$$

Finally, combining (5.33) and (5.36) implies that $\left(\lambda^{*}, \beta^{*}\right) \in \Lambda_{y}^{o}(\bar{x}, \bar{y})$, provided that $\lambda^{*} \geq 0$. This concludes the proof of the proposition.

6. Applications to necessary optimality conditions in the original optimistic model. The concluding section of the paper is devoted to applications of the above sensitivity results to deriving new necessary optimality conditions for the original optimistic formulation $\left(\mathrm{P}_{o}\right)$ in bilevel programming. In fact we establish certain stationarity conditions of various types among which are of those types known for more conventional auxiliary optimistic formulation $(\mathrm{P})$ together with stationarity conditions of the novel types for $\left(\mathrm{P}_{o}\right)$.

For the reader's convenience, we recall that $S$ and $S_{o}$ refer to the solution maps of the lower-level (1.5) and upper-level (1.6) problems, respectively, and that the lowerlevel Lagrange function $\mathcal{L}$ and Lagrange multipliers set $\Lambda(\bar{x}, \bar{y})$ are given by (5.2). Also the index sets $\eta, \theta$, and $\nu$ of a major interest here are defined in (4.4).

We start with stationarity notions of the new "KM" and "KN" types for the original optimistic bilevel program $\left(\mathrm{P}_{o}\right)$ reflecting the difference between the KKT-type optimality conditions obtained via the inner semicontinuity and inner semicompactness, respectively, of the optimal solution map $S_{o}$ for the upper-level problem.

Definition 6.1 (KM-stationarity for original optimistic bilevel programs). A point $\bar{x}$ is $\mathrm{P}_{o}$-KM-stationary if we can find some $\bar{y} \in S_{o}(\bar{x})$ such that there exist $(\alpha, \beta) \in \mathbb{R}^{k+p}, \lambda \in \mathbb{R}_{+},\left(\mu_{s}, v_{s}\right) \in \mathbb{R}^{k+1}$, and $y_{s} \in S(\bar{x})$ as $s=1, \ldots, n+1$ satisfying

$$
\begin{aligned}
\nabla_{x} F(\bar{x}, \bar{y})+\nabla G(\bar{x})^{\top} \alpha+\lambda\left(\nabla_{x} f(\bar{x}, \bar{y})-\sum_{s=1}^{n+1} v_{s} \nabla_{x} f\left(\bar{x}, y_{s}\right)\right) & \\
+\sum_{i=1}^{p} \beta_{i} \nabla_{x} g_{i}(\bar{x}, \bar{y})-\lambda \sum_{s=1}^{n+1} v_{s} \sum_{i=1}^{p} \mu_{i s} \nabla_{x} g_{i}\left(\bar{x}, y_{s}\right) & =0, \\
\nabla_{y} F(\bar{x}, \bar{y})+\lambda \nabla_{y} f(\bar{x}, \bar{y})+\sum_{i=1}^{p} \beta_{i} \nabla_{y} g_{i}(\bar{x}, \bar{y}) & =0, \\
\forall s=1, \ldots, n+1, \nabla_{y} f\left(\bar{x}, y_{s}\right)+\sum_{i=1}^{p} \mu_{i s} \nabla_{y} g_{i}\left(\bar{x}, y_{s}\right) & =0, \\
\forall s=1, \ldots, n+1, i=1, \ldots, p, \mu_{i s} \geq 0, \mu_{i s} g_{i}\left(\bar{x}, y_{s}\right) & =0, \\
\forall j=1, \ldots, k, \alpha_{j} \geq 0, \alpha_{j} G_{j}(\bar{x}) & =0, \\
\forall i=1, \ldots, p, \beta_{i} \geq 0, \beta_{i} g_{i}(\bar{x}, \bar{y}) & =0, \\
\forall s=1, \ldots, n+1, v_{s} \geq 0, \sum_{s=1}^{n+1} v_{s} & =1 .
\end{aligned}
$$

Relationships (6.1)-(6.7) are called the KM-stationarity conditions.

Definition 6.2 (KN-stationarity for original optimistic bilevel programs). A point $\bar{x}$ is $\mathrm{SP}_{o}-\mathrm{KN}$-stationary (resp., $\mathrm{P}_{o}-\mathrm{KN}$-stationary) if for every $\bar{y} \in S_{o}(\bar{x})$ (resp., 
for some $\left.\bar{y} \in S_{o}(\bar{x})\right)$ there exist $(\alpha, \beta, \mu) \in \mathbb{R}^{k+2 p}$ and $\lambda \in \mathbb{R}_{+}$such that relationships (6.2) and (6.5)-(6.6) hold along with

$$
\begin{aligned}
\nabla_{x} F(\bar{x}, \bar{y})+\sum_{j=1}^{k} \alpha_{j} \nabla G_{j}(\bar{x})+\sum_{i=1}^{p}\left(\beta_{i}-\lambda \mu_{i}\right) \nabla_{x} g_{i}(\bar{x}, \bar{y}) & =0, \\
\nabla_{y} f(\bar{x}, \bar{y})+\sum_{i=1}^{p} \mu_{i} \nabla_{y} g_{i}(\bar{x}, \bar{y}) & =0 \\
\forall i=1, \ldots, p, \mu_{i} \geq 0, \mu_{i} g_{i}(\bar{x}, \bar{y}) & =0 .
\end{aligned}
$$

All the relationships (6.2), (6.5)-(6.6), and (6.8)-(6.10) considered together are called the KN-stationarity conditions.

Observe that the stationarity concepts of Definitions 6.1 and 6.2 arise from the LLVF reformulation of the original problem $\left(\mathrm{P}_{o}\right)$. Next we introduce stationarity concepts related to the OPCC and OPEC reformulations of $\left(\mathrm{P}_{o}\right)$.

Definition 6.3 (M-stationarity for original optimistic bilevel programs). A point $\bar{x}$ is $\mathrm{SP}_{o}$-M-stationary (resp., $\mathrm{P}_{o}$-M-stationary) if for every $(\bar{y}, \bar{u}) \in S_{o}^{h}(\bar{x})$ (resp., for some $\left.(\bar{y}, \bar{u}) \in S_{o}^{h}(\bar{x})\right)$ there exists a triple $(\alpha, \beta, \gamma) \in \mathbb{R}^{k+p+m}$ such that relationships (4.6)-(4.7) and (6.5) hold along with

$$
\begin{array}{r}
\nabla_{x} F(\bar{x}, \bar{y})+\nabla G(\bar{x})^{\top} \alpha+\nabla_{x} g(\bar{x}, \bar{y})^{\top} \beta+\nabla_{x} \mathcal{L}(\bar{x}, \bar{y}, \bar{u})^{\top} \gamma=0 \\
\nabla_{y} F(\bar{x}, \bar{y})+\nabla_{y} g(\bar{x}, \bar{y})^{\top} \beta+\nabla_{y} \mathcal{L}(\bar{x}, \bar{y}, \bar{u})^{\top} \gamma=0 .
\end{array}
$$

All the relationships (4.6)-(4.7), (6.5), and (6.11)-(6.12) considered together are called the M-stationarity conditions.

Similarly to M-stationarity, we define C-stationarity (resp., S-stationarity) by replacing (4.7) with (5.11) and (5.13), respectively. Note that for any $A:=K N, M, C, S$ the term "SP ${ }_{o}$-A-stationary" stands for strong $\mathrm{P}_{o}$-A-stationary. The following diagram provides links between these stationarity concepts:

$$
\begin{aligned}
& \begin{array}{ccccc}
\mathrm{SP}_{o} \text {-M-stationary } & \Longleftarrow & \mathrm{SP}_{o} \text {-S-stationary } & \Longrightarrow & \mathrm{SP}_{o} \text {-C-stationary } \\
\Downarrow & \Downarrow & & \Downarrow \\
\mathrm{P}_{o} \text {-M-stationary } & \Longleftarrow & \mathrm{P}_{o} \text {-S-stationary } & \Longrightarrow & \mathrm{P}_{o} \text {-C-stationary }
\end{array} \\
& \text { (2) } \Downarrow \\
& \mathrm{SP}_{o} \text {-KN-stationary } \Longrightarrow \mathrm{P}_{o} \text {-KN-stationary }(\lambda \text { free }) \stackrel{(1)}{\Longleftrightarrow} \mathrm{P}_{o} \text {-KM-stationary }
\end{aligned}
$$

where assumptions (1) and (2) are formulated as

$$
\left\{\begin{array}{l}
(1) \quad S(\bar{x})=\{\bar{y}\}, \Lambda(\bar{x}, \bar{y})=\{\mu\}, \\
(2) \quad \sum_{l=1}^{m} \gamma_{l} \nabla_{x, y} \mathcal{L}_{l}(\bar{x}, \bar{y}, \bar{u})=0 .
\end{array}\right.
$$

The proof of this chain of implications follows along the lines of that in [64, Theorem 3.1.9], where a similar diagram is constructed for the stationarity concepts tailored to the conventional optimistic bilevel program (P). Note that the term " $\lambda$ free" used in the above diagram is valid only for implication " $\mathrm{P}_{o^{-}}$S-stationary $\stackrel{(2)}{\Longrightarrow}$ $\mathrm{P}_{o}$-KN-stationary ( $\lambda$ free)," where it refers to the fact that the multiplier $\lambda$ must not be nonnegative as in the case in Definition 6.2.

Now we are ready to establish another important result of the paper on justifying the above stationarity conditions for the original optimistic bilevel program $\left(\mathrm{P}_{o}\right)$. They are based on sensitivity analysis of the two-level value function obtained in the 
previous section combined with fundamental calculus rules of generalized differentiation. To proceed, recall that a point $\bar{x} \in X:=\left\{x \in \mathbb{R}^{n} \mid G(x) \leq 0\right\}$ is upper-level regular if it holds that

$$
\left[\nabla G(\bar{x})^{\top} \alpha=0, \alpha \geq 0, \alpha^{\top} G(\bar{x})=0\right] \Longrightarrow \alpha=0,
$$

which is the dual form of the classical MFCQ for the upper-level constraints.

THEOREM 6.4 (justification of KM-stationarity for original optimistic bilevel programs). Let $\bar{x}$ be an upper-level regular local optimal solution to $\left(P_{o}\right)$, let $S_{o}$ from (5.30) be inner semicompact at $\bar{x}$, and let the lower-level regularity (4.2) be satisfied at all $(\bar{x}, \bar{y})$ with $\bar{y} \in S(\bar{x})$. Suppose furthermore that $\left(A_{1}^{v}\right)$ and $\left(A_{2}^{v}\right)$ are satisfied at all $(\bar{x}, \bar{y})$ with $\bar{y} \in S_{o}(\bar{x})$. Then the point $\bar{x}$ is $P_{o}-K M$-stationary.

Proof. It follows from Theorem 5.11(ii) that the two-level value function $\varphi_{o}$ is Lipschitz continuous around $\bar{x}$. Thus $\partial \varphi_{o}(\bar{x}) \neq \emptyset$ while $\partial^{\infty} \varphi_{o}(\bar{x})=\{0\}$, and the qualification condition (2.12) of Theorem 2.2 holds at $\bar{x}$. Employing now the optimality condition (2.11) of the latter theorem with the well-known formula

$$
N_{X}(\bar{x})=\left\{\nabla G(\bar{x})^{\top} \alpha \mid \alpha \geq 0, \alpha^{\top} G(\bar{x})=0\right\},
$$

valid under the assumed upper-level regularity of $\bar{x}$, and then taking into account that the set on the right-hand side of the inclusion in Theorem 5.11(ii) is nonempty, we arrive at the $\mathrm{P}_{o}$-KM-stationarity.

THEOREM 6.5 (justification of KN-stationarity for original optimistic bilevel programs). Let $\bar{x}$ be an upper-level regular local optimal solution to $\left(\mathrm{P}_{o}\right)$. Then we have the following:

(i) Assume that for all $\bar{y} \in S_{o}(\bar{x})$ the inner semicontinuity of the mapping $S_{o}$ in (5.30), the lower-level regularity (4.2), and conditions $\left(A_{1}^{v}\right)$ and $\left(A_{2}^{v}\right)$ are all satisfied at $(\bar{x}, \bar{y})$. Then $\bar{x}$ is $S P_{o}-K N$-stationary.

(ii) If all the assumptions in (i) are satisfied at some point $(\bar{x}, \bar{y})$ with $\bar{y} \in S_{o}(\bar{x})$, then $\bar{x}$ is $P_{o}-K N$-stationary.

Proof. To justify (i), observe that the inclusion in Theorem 5.11(i) depends on a point $(\bar{x}, \bar{y}) \in \operatorname{gph} S_{o}$, where $S_{o}$ is inner semicontinuous. Thus if this inclusion is satisfied at every $(\bar{x}, \bar{y}) \in \operatorname{gph} S_{o}$ and all the other qualification conditions of Theorem 5.11(i) hold at these points, then the result follows as in the proof of Theorem 6.4. Assertion (ii) is obtained by combining Theorem 2.2 and Theorem 5.11(i).

Next we address the $M$-stationarity conditions for the original bilevel program.

THEOREM 6.6 (justification of M-stationarity for original optimistic bilevel programs). Let $\bar{x}$ be an upper-level regular local optimal solution to $\left(\mathrm{P}_{o}\right)$, where the lower-level problem (1.2) is convex. Assume that the lower-level regularity is satisfied at all $(\bar{x}, \bar{y})$ with $\bar{y} \in S(\bar{x})$, while $\left(A_{1}^{m}\right)$ and $\left(A_{2}^{m}\right)$ are satisfied at $(\bar{x}, \bar{y}, \bar{u})$ for all $(\bar{y}, \bar{u}) \in S_{o}^{h}(\bar{x})$. The following hold:

(i) If the solution map $S_{o}^{h}$ in (5.5) is inner semicontinuous at $(\bar{x}, \bar{y}, \bar{u})$ for all $(\bar{y}, \bar{u}) \in S_{o}^{h}(\bar{x})$, then $\bar{x}$ is $S P_{o}-M$-stationary.

(ii) If $S_{o}^{h}$ is inner semicompact at $\bar{x}$, then $\bar{x}$ is $P_{o}$-M-stationary.

Proof. The proof follows similarly to that of the previous theorem by employing Theorem 2.2 and Theorem 5.2(i) and (ii), respectively.

Another way to derive the $\mathrm{P}_{o}$-M-stationarity is by using the upper estimate of $\partial \varphi_{o}(\bar{x})$ obtained via the OPEC reformulation of the two-level value function (5.15); see Corollary 5.5(ii). Note also that if the inner semicontinuity and qualification conditions $\left(A_{1}^{m}\right)$ and $\left(A_{2}^{m}\right)$ are satisfied only at one point $(\bar{x}, \bar{y}, \bar{u})$ in Theorem 6.6 , we 
still can get $\mathrm{P}_{o}$-M-stationarity whenever the reference couple $(\bar{y}, \bar{u}) \in S_{o}^{h}(\bar{x})$ is known a priori. A similar comment can be made for $\mathrm{P}_{o}-\mathrm{KN}$-stationarity.

Proceeding as in the proof of Theorem 6.6, C-stationarity (resp., S-stationarity) for a local optimal solution to $\left(\mathrm{P}_{o}\right)$ can be derived by combining Theorem 2.2 and Theorem 5.3 (resp., Theorem 5.4). The next example illustrates Theorem 6.6.

Example 6.7 (numerical illustration of qualification and stationarity conditions for $\left.\left(\mathrm{P}_{o}\right)\right)$. Consider the original optimistic bilevel program from [14, Example 4.1]:

$$
\min _{x \in \mathbb{R}_{+}}\left\{\min _{y \in \mathbb{R}}\left\{x^{2}+y^{2} \mid y \in S(x):=\arg \min \{x y+y \mid y \geq 0\}\right\}\right\} .
$$

The KKT reformulation of the corresponding two-level value function is

$$
\varphi_{o}(x):=\min _{y, u}\left\{x^{2}+y^{2} \mid x-u+1=0, u \geq 0, y \geq 0, u y=0\right\}= \begin{cases}x^{2} & \text { if } x \geq-1 \\ \infty & \text { otherwise }\end{cases}
$$

It is obvious that $\bar{x}=0$ is the unique optimal solution to this program and that $\varphi_{o}$ is smooth near $\bar{x}$. We have $S_{o}^{h}(x)=\{(0, x+1)\}$ if $x \geq-1$ and $S_{o}^{h}(x)=\emptyset$ otherwise, and hence $S_{o}^{h}$ is a single-valued and continuous mapping on its graph. Furthermore, $\Lambda_{y}^{e m}(\bar{x}, \bar{y}, \bar{u}, 0)=\{0\} \times \mathbb{R}$ if $(\bar{x}, \bar{y}, \bar{u})=(-1,0,0)$ and $\Lambda_{y}^{e m}(\bar{x}, \bar{y}, \bar{u}, 0)=\{(0,0)\}$ for all the other points of $g p h S_{o}^{h}$. From the observations made in sections 3 and 4 , this implies that the corresponding constraint qualifications $\left(A_{1}^{m}\right)$ and $\left(A_{2}^{m}\right)$ are satisfied at all points of the graph of $S_{o}^{h}$ except $(-1,0,0)$, which is not optimal.

It is worth mentioning that the upper-level regularity (6.13) in the results above can be replaced by the weaker calmness property of the mapping $v \rightrightarrows\left\{x \in \mathbb{R}^{n} \mid G(x)+\right.$ $v \leq 0\}$, which is automatic if $G$ is a linear function. Furthermore, as mentioned previously in subsection 5.1 , the qualification condition $\left(A_{1}^{m}\right)$ can also be replaced by the weaker calmness property of the mapping $\Psi$ in (5.16) that holds, in particular, if both functions $g$ and $(x, y) \mapsto \nabla_{y} f(x, y)$ are linear.

Remark 6.8 (on Lipschitz continuity of the two-level value function $\varphi_{o}$ ). We can see from the proof of Theorem 6.6 that the local Lipschitz continuity of $\varphi_{o}$ was used twice: to ensure the nonemptiness of $\partial \varphi_{o}(\bar{x})$ and the application of the optimality condition (2.11) of Theorem 2.2. Observe to this end that the Lipschitz property of $\varphi_{o}$ is not needed for bilevel programs without upper-level constraints (i.e., if $X:=\mathbb{R}^{n}$ ); in this case the qualification condition (2.12) holds automatically and also allows us to drop assumption $\left(A_{2}^{m}\right)$ in Theorem 6.6. However, we still have to make sure that $\partial \varphi_{o}(\bar{x}) \neq \emptyset$, which happens in many non-Lipschitzian situations; see, e.g., [38, 39, 43, $51]$.

The rest of this section is devoted to the relationship between the original $\left(\mathrm{P}_{o}\right)$ and conventional $(\mathrm{P})$ versions in optimistic bilevel programming. We begin with noting that both problems are equivalent from the viewpoint of global optimal solutions. The next proposition clarifies the relationship between them for local solutions.

Proposition 6.9 (relationship between local optimal solutions to original and conventional optimistic programs). The following assertions hold:

(i) Let $\bar{x}$ be a local optimal solution to $\left(\mathrm{P}_{o}\right)$, then $(\bar{x}, \bar{y})$ for any $\bar{y} \in S_{o}(\bar{x})$ is a local optimal solution to $(\mathrm{P})$.

(ii) Let $(\bar{x}, \bar{y})$ with some $\bar{y} \in S_{o}(\bar{x})$ be a local optimal solution to $(\mathrm{P})$. If $S_{o}$ is inner semicontinuous at $(\bar{x}, \bar{y})$, then $\bar{x}$ is a local optimal solution to $\left(\mathrm{P}_{o}\right)$.

Proof. To justify (i), assume that $(\bar{x}, \bar{y})$ for some $\bar{y} \in S_{o}(\bar{x})$ is not a local optimal solution to $(\mathrm{P})$. Then we can find a sequence $\left(x^{k}, y^{k}\right)$ with $x^{k} \rightarrow \bar{x}, y^{k} \rightarrow \bar{y}$ and $x^{k} \in X, y^{k} \in S\left(x^{k}\right)$ such that we have $F\left(x^{k}, y^{k}\right)<F(\bar{x}, \bar{y})=\varphi_{o}(\bar{x})$ for all $k$. By 
the definition of $\varphi_{o}$, it follows that $\varphi_{o}\left(x^{k}\right) \leq F\left(x^{k}, y^{k}\right)$, for all $k$. Thus we have $\varphi_{o}\left(x^{k}\right)<\varphi_{o}(\bar{x}), x^{k} \in X$ for all $k$. This completes the proof of (i) given that $\bar{x} \in X$.

To proceed with (ii), assume that $\bar{x}$ is not a local optimal solution of $\left(\mathrm{P}_{o}\right)$, while all the other hypotheses of (ii) are satisfied. We can find a sequence $x^{k} \rightarrow \bar{x}\left(x^{k} \in X\right)$ such that $\varphi_{o}\left(x^{k}\right)<\varphi_{o}(\bar{x})$ for all $k$. $S_{o}$ being inner semicontinuous at $(\bar{x}, \bar{y})$, there is a sequence $y^{k} \in S_{o}\left(x^{k}\right)$ with $y^{k} \rightarrow \bar{y}$. Considering the definition of $S_{o}$ (1.6), we have $y^{k} \in$ $S\left(x^{k}\right)$. Further observe that with $y^{k} \in S_{o}\left(x^{k}\right)$, we have $F\left(x^{k}, y^{k}\right)=\varphi_{o}\left(x^{k}\right)$ for all $k$. Hence, it follows that $F\left(x^{k}, y^{k}\right)<F(\bar{x}, \bar{y}), x^{k} \in X, y^{k} \in S\left(x^{k}\right)$ for all $k$, given that $\varphi_{o}\left(x^{k}\right)<\varphi_{o}(\bar{x})$ for all $k$. This contradicts the fact that $(\bar{x}, \bar{y})$ is a local optimal solution of problem (P), given that $x^{k} \rightarrow \bar{x}, y^{k} \rightarrow \bar{y}$.

Recall that assertion (i) of Proposition 6.9 was initially proved in [17] under the upper semicontinuity of the lower-level solution map $S$ from (1.5) and then in [11] under the uniform boundedness of $S$. As we show now, no assumption is in fact needed. However, the next example demonstrates that for the converse implication (ii), the inner semicontinuity of the mapping $S_{o}$ in (1.6) is essential.

Example 6.10 (failure of Proposition 6.9(ii) with no inner semicontinuity of $S_{o}$ ). Consider the following bilevel program taken from [8, section 5.5]:

$$
\text { "min" }\left\{x \mid x \in[-1,1], y \in S(x):=\arg \min _{y}\{x y \mid y \in[0,1]\} .\right.
$$

The lower-level solution map is calculated by

$$
S(x)=\left\{\begin{array}{lll}
{[0,1]} & \text { if } & x=0, \\
\{0\} & \text { if } & x>0, \\
\{1\} & \text { if } & x<0 .
\end{array}\right.
$$

It is easy to see that $(\bar{x}, \bar{y})=(0,0)$ is a local optimal solution to the conventional optimistic model $(\mathrm{P})$, but $\bar{x}=0$ is not a local optimal solution to the corresponding original optimistic formulation $\left(\mathrm{P}_{o}\right)$. Note that in this case we have $S_{o}(x)=S(x)$ for all $x \in X:=[-1,1]$. Moreover, $-1 / 2^{k} \rightarrow 0$ while $S_{o}\left(-1 / 2^{k}\right)=S\left(-1 / 2^{k}\right)=\{1\}$ for all $k$, but $1 \neq 0$. Thus $S_{o}$ is not inner semicontinuous at $(0,0)$.

The next result establishes the link between the stationarity conditions for the original optimistic formulation $\left(\mathrm{P}_{o}\right)$ defined at the beginning of this section and those for the conventional/auxiliary optimistic problem (P) that we introduce now.

Definition 6.11 (KM-, KN-, and M-stationarity concepts for conventional optimistic bilevel programs). Let $(\bar{x}, \bar{y})$ be feasible to $(\mathrm{P})$. We say that it is

(i) P-KM-stationary if there are $(\alpha, \beta) \in \mathbb{R}^{k+p}, \lambda \in \mathbb{R}_{+},\left(\mu_{s}, v_{s}\right) \in \mathbb{R}^{k+1}$, and $y_{s} \in S(\bar{x}), s=1, \ldots, n+1$, such that the KM-stationarity conditions (6.1)-(6.7) hold;

(ii) P-KN-stationary if there are $(\alpha, \beta, \mu) \in \mathbb{R}^{k+2 p}$ and $\lambda \in \mathbb{R}_{+}$such that the $K N$-stationarity conditions (6.2), (6.5)-(6.6), and (6.8)-(6.10) hold;

(iii) P-M-stationary if there are $(\alpha, \beta, \gamma) \in \mathbb{R}^{k+p+m}$ such that the M-stationarity conditions (4.6)-(4.7), (6.5), and (6.11)-(6.12) hold.

Similarly to (iii) the C- and S-stationarity concepts can be defined for problem (P) via the corresponding notions tailored to the original optimistic problem $\left(\mathrm{P}_{o}\right)$; cf. Definition 6.3 and the related discussion. Stronger versions of the KN-, M-, C- and Sstationarity conditions have also been derived in the literature; see [14, 64], but we do not consider them here since relationships to their $\mathrm{P}_{o}$ counterparts will be investigated separately in our future research. As already mentioned in section 1, problem $(\mathrm{P})$ has been intensively studied in the literature. In particular, the KM- and KN-stationarity conditions have been derived under various assumptions in $[11,13,15,16,41,61]$. For the other conditions, see, e.g., [14, 20,64] and the references therein. 
THEOREM 6.12 (relationship between stationarity conditions for the conventional and original optimistic bilevel programs). The following assertions hold:

(i) A point $\bar{x}$ is $P_{o}-K M$-stationary (resp., $P_{o}-K N$-stationary) if and only if there exists $\bar{y} \in S_{o}(\bar{x})$ such that $(\bar{x}, \bar{y})$ is $P$-KM-stationary (resp., $P$-KN-stationary).

(ii) A point $\bar{x}$ is $P_{o}$-M-stationary (resp., $P_{o}-C, P_{o}$-S-stationary) if and only if there exists $(\bar{y}, \bar{u}) \in S_{o}^{h}(\bar{x})$ such that $(\bar{x}, \bar{y})$ is $P$-M-stationary (resp., $P$-C, $P$-S-stationary).

Proof. The proof follows directly by comparing the necessary optimality/stationarity conditions obtained above in Theorems $6.4,6.5$, and 6.6 for the original bilevel formulation $\left(\mathrm{P}_{o}\right)$ and the ones for problem $(\mathrm{P})$ from Definition 6.11.

A natural question arises when comparing the constraint qualifications for the corresponding stationarity in $(\mathrm{P})$ and $\left(\mathrm{P}_{o}\right)$. The constraint qualifications developed above to derive stationarity conditions for $\left(\mathrm{P}_{o}\right)$ occur to be stronger than those needed for (P) (cf. $[11,13,14,15,16,20,41,58,61,64]$ ) when the Lipschitz continuity of $\varphi_{o}$ (5.1) is involved; cf. the discussion in Remark 6.8. However, putting aside the inner semicontinuity/semicompactness, the assumptions we introduce in the context of $\left(\mathrm{P}_{o}\right)$ to get $\mathrm{P}_{o}-\mathrm{KM}-, \mathrm{P}_{o}-\mathrm{KN}-, \mathrm{P}_{o^{-}} \mathrm{M}-, \mathrm{P}_{o^{-}} \mathrm{C}-$, and $\mathrm{P}_{o^{-}}$-S-stationarity are closely related to those used for $(\mathrm{P})$ in the aforementioned references, in particular, if there are no upper-level constraints. Also observe that the implication in Proposition 6.9(i) opens a new perspective to derive stationarity conditions for the original optimistic bilevel program via the auxiliary problem $(\mathrm{P})$ with less restrictive assumptions.

Finally, we mention that the sensitivity results for two-level value functions obtained in section 5 can readily be applied for sensitivity analysis of the auxiliary problem $(\mathrm{P})$ and also to deriving necessary optimality conditions for the pessimistic bilevel program $\left(\mathrm{P}_{p}\right)$; see [12] for the recent developments in the latter case.

Acknowledgments. The authors are indebted to two anonymous referees and the handling Associate Editor Jiří Outrata for their constructive remarks, which allowed us to improve the original presentation. We are also grateful to René Henrion for his suggestions on the calmness property discussed in Remark 4.2.

\section{REFERENCES}

[1] W. Achtziger And C. Kanzow, Mathematical programs with vanishing constraints: Optimality conditions and constraint qualifications, Math. Program., 114 (2008), pp. 69-99.

[2] J. F. BARD, Practical Bilevel Optimization: Algorithms and Applications, Kluwer Academic, Dordrecht, The Netherlands, 1998.

[3] J. F. Bonnans and A. Shapiro, Perturbation Analysis of Optimization Problems, Springer, New York, 2000.

[4] M. Ĉervinka, C. Matonoha, and J. V. Outrata, On the computation of relaxed pessimistic solutions to MPEC, Optim. Method Softw. (2012), to appear.

[5] F. H. Clarke, Optimization and Nonsmooth Analysis, Wiley, New York, 1983.

[6] B. Colson, P. Marcotte, and G. Savard, An overview of bilevel optimization, Ann. Oper. Res., 153 (2007), pp. 235-256.

[7] S. Dassanayaka, Methods of Variational Analysis in Pessimistic Bilevel Programming, Ph.D. thesis (B. S. Mordukhovich advisor), Department of Mathematics, Wayne State University, Detroit, MI, 2010.

[8] S. Dempe, Foundations of Bilevel Programming, Kluwer Academic, Dordrecht, The Netherlands, 2002.

[9] S. Dempe, N. Dinh, And J. Dutta, Optimality conditions for a simple convex bilevel programming problem, in Variational Analysis and Generalized Differentiation in Optimization and Control, R. S. Burachik and J.-C. Yao, eds., Springer Optim. Appl. S47, Springer, Berlin, 2010, pp. 149-161.

[10] S. Dempe And J. DutTa, Is bilevel programming a special case of mathematical programming with equilibrium constraints?, Math. Program., 131 (2012), pp. 37-48. 
[11] S. Dempe, J. Dutta, and B. S. Mordukhovich, New necessary optimality conditions in optimistic bilevel programming, Optimization, 56 (2007), pp. 577-604.

[12] S. Dempe, B. S. Mordukhovich, and A. B. Zemконo, Necessary optimality conditions in pessimistic bilevel programming, Optimization, to appear.

[13] S. Dempe And A. B. Zemконo, The generalized Mangasarian-Fromowitz constraint qualification and optimality conditions for bilevel programs, J. Optim. Theory Appl., 148 (2011), pp. 433-441.

[14] S. Dempe And A. B. Zемконо, On the Karush-Kuhn-Tucker reformulation of the bilevel optimization problem, Nonlinear Anal., 75 (2012), pp. 1202-1218.

[15] S. Dempe And A. B. Zемконо, The bilevel programming problem: Reformulations, constraint qualifications and optimality conditions, Math. Program., to appear.

[16] N. Dinh, B. S. Mordukhovich, And T. T.A. NGhia, Subdifferentials of value functions and optimality conditions for DC and bilevel infinite and semi-infinite programs, Math. Program., 123 (2010), pp. 101-138.

[17] J. Dutta And S. Dempe, Bilevel programming with convex lower level problems, in Optimization with Multivalued Mappings, S. Dempe and V. Kalashnikov, eds., Springer Optim. Appl. 2, Springer, New York, 2006, pp. 51-71.

[18] M. C. Ferris, Weak Sharp Minima and Penalty Functions in Mathematical Programming, Ph.D. thesis, Churchill College, University of Cambridge, Cambridge, UK, 1989.

[19] A. V. Fiacco, Introduction to Sensitivity and Stability Analysis in Nonlinear Programming, Academic Press, New York, 1983.

[20] M. L. Flegel, Constraint Qualification and Stationarity Concepts for Mathematical Programs with Equilibrium Constraints, Ph.D. thesis (C. Kanzow, advisor), Institute of Applied Mathematics and Statistics, University of Würzburg, Würzburg, 2005.

[21] M. L. Flegel and C. Kanzow, A direct proof for $M$-stationarity under MPEC-GCQ for mathematical programs with equilibrium constraints, in Optimization with Multivalued Mappings, S. Dempe and V. Kalashnikov, eds., Springer Optim. Appl. 2, Springer, New York, 2006, pp. 111-122.

[22] M. L. Flegel, C. Kanzow, and J. V. Outrata, Optimality conditions for disjunctive programs with application to mathematical programs with equilibrium constraints, J. SetValued Anal., 15 (2007), pp. 139-162.

[23] J. Gauvin and F. Dubeau, Differential properties of the marginal function in mathematical programming, Math. Programming Stud., 18 (1982), pp. 101-119.

[24] R. Henrion, A. Jourani, AND J. Outrata, On the calmness of a class of multifunctions, SIAM J. Optim., 13 (2002), pp. 603-618.

[25] R. Henrion and J. V. Outrata, A subdifferential condition for calmness of multifunctions, J. Math. Anal. Appl., 258 (2001), pp. 110-130.

[26] R. Henrion, J. V. Outrata, and T. Surowiec, On the coderivative of normal cone mappings for inequality systems, Nonlinear Anal., 71 (2009), pp. 1213-1226.

[27] R. Henrion and T. Surowiec, On calmness conditions in convex bilevel programming, Appl. Anal., 90 (2011), pp. 951-970.

[28] T. Hoheisel, C. Kanzow, and J. V. Outrata, Exact penalty results for mathematical programs with vanishing constraints, Nonlinear Anal., 72 (2010), pp. 2514-2526.

[29] X. Hu AND D. RALPH, A note on sensitivity of value functions of mathematical programs with complementarity constraints, Math. Program., 93 (2002), pp. 265-279.

[30] S. LoHsE, Eine spezielle klasse von zwei-ebenen-optimierungsaufgaben, Ph.D. thesis (S. Dempe, advisor), Fakultät für Mathematik und Informatik, TU Bergakademie Freiberg, Freiberg, 2010.

[31] S. Lu, Implications of the constant rank constraint qualification, Math. Program., 126 (2011), pp. 365-392.

[32] Y. LuCET AND J. J. YE, Sensitivity analysis of the value function for optimization problems with variational inequality constraints, SIAM J. Control Optim., 40 (2001), pp. 699-723. (with erratum in SIAM J. Control Optim., 41 (2002), pp. 1315-1319).

[33] Z. Q. LuO, J.-S. PAng, And D. RAlPh, Mathematical Programs with Equilibrium Constraints, Cambridge University Press, Cambridge, UK, 1996.

[34] O. L. Mangasarian and S. Fromovitz, The Fritz John necessary optimality conditions in the presence of equality and inequality constraints, J. Math. Anal. Appl., 17 (1967), pp. $37-47$.

[35] B. S. MordukHovich, Maximum principle in problems of time optimal control with nonsmooth constraints, J. Appl. Math. Mech., 40 (1976), pp. 690-669.

[36] B. S. MordukHovich, Metric approximations and necessary optimality conditions for general classes of extremal problems, Soviet Math. Dokl., 22 (1980), pp. 526-530.

Copyright $@$ by SIAM. Unauthorized reproduction of this article is prohibited. 
[37] B. S. Mordukhovich, Nonsmooth analysis with nonconvex generalized differentials and conjugate mappings, Dokl. Akad. Nauk BSSR, 28 (1984), pp. 976-979 (in Russian).

[38] B. S. Mordukhovich, Variational Analysis and Generalized Differentiation. I. Basic Theory, Springer, Berlin, 2006.

[39] B. S. Mordukhovich, Variational Analysis and Generalized Differentiation. II. Applications, Springer, Berlin, 2006.

[40] B. S. Mordukhovich AND N. M. NAM, Variational stability and marginal functions via generalized differentiation, Math. Oper. Res., 30 (2005), pp. 800-816.

[41] B. S. Mordukhovich, M. N. Nam, And H. M. Phan, Variational analysis of marginal functions with applications to bilevel programming, J. Optim. Theory Appl., 152 (2011), pp. $557-586$.

[42] B. S. Mordukhovich, N. M. NAm, And N. D. Yen, Fréchet subdifferential calculus and optimality conditions in nondifferentiable programming, Optimization, 55 (2006), pp. 685-708.

[43] B. S. Mordukhovich, M. N. Nam, And N. D. Yen, Subgradients of marginal functions in parametric mathematical programming, Math. Program., 116 (2009), pp. 369-396.

[44] B. S. Mordukhovich And J. V. Outrata, Coderivative analysis of quasi-variational inequalities with applications to stability and optimization, SIAM J. Optim., 18 (2007), 389-412.

[45] J. V. Outrata, On the numerical solution of a class of Stackelberg problems, Z. Oper. Res., 34 (1990), pp. 255-277.

[46] J. V. OUtrata, Optimality conditions for a class of mathematical programs with equilibrium constraints, Math. Oper. Res., 24 (1999), pp. 627-644.

[47] J. V. Outrata, M. Kočvara, And J. Zowe, Nonsmooth Approach to Optimization Problems with Equilibrium Constraints, Kluwer Academic, Dordrecht, The Netherlands, 1998.

[48] B. T. Polyak, Sharp Minima, Institute of Control Sciences Lecture Notes, Moscow, USSR, 1979; presented at the IIASA Workshop on Generalized Lagrangians and Their Applications, IIASA, Laxenburg, Austria, 1979.

[49] S. M. Robinson, Some continuity properties of polyhedral multifunctions, Math. Programming Stud., 14 (1981), pp. 206-214.

[50] R. T. RockAfellar, Marginal values and second-order necessary conditions for optimality, Math. Program., 26 (1983), pp. 245-286.

[51] R. T. Rockafellar and R. J. B. Wets, Variational Analysis, Springer, Berlin, 1998.

[52] H. Scheel And S. Scholtes, Mathematical programs with complementarity constraints: Stationarity, optimality, and sensitivity, Math. Oper. Res., 25 (2000), pp. 1-22.

[53] W. Schirotzeк, Nonsmooth Analysis, Springer, Berlin, 2007.

[54] W. E. SChmitendorf, A simple derivation of necessary conditions for static minmax problems, J. Math. Anal. Appl., 70 (1979), pp. 486-489.

[55] K. Shimizu, Y. IshizukA, And J. F. BARD, Nondifferentiable and Two-Level Mathematical Programming, Kluwer Academic, Dordrecht, The Netherlands, 1997.

[56] J. J. Ye, New uniform parametric error bounds, J. Optim. Theory Appl., 98 (1998), pp. 197219.

[57] J. J. YE, Optimality conditions for optimization problems with complementarity constraints, SIAM J. Optim., 9 (1999), pp. 374-387.

[58] J. J. YE, Nondifferentiable multiplier rules for optimization and bilevel optimization problems, SIAM J. Optim., 15 (2004), pp. 252-274.

[59] J. J. YE, Necessary and sufficient optimality conditions for mathematical programs with equilibrium constraints, J. Math. Anal. Appl., 307 (2005), pp. 350-369.

[60] J. J. YE AND X. Y. YE, Necessary optimality conditions for optimization problems with variational inequality constraints, Math. Oper. Res., 22 (1997), pp. 977-997.

[61] J. J. Ye AND D. L. ZHU, Optimality conditions for bilevel programming problems, Optimization, 33 (1995), pp. 9-27 (with erratum in Optimization, 39 (1997), pp. 361-366).

[62] J. J. Ye AND D. L. ZHU, New necessary optimality conditions for bilevel programs by combining the MPEC and value function approaches, SIAM J. Optim., 20 (2010), pp. 1885-1905.

[63] А. B. Zемконо, A simple approach to optimality conditions in minmax programming, Optimization, to appear.

[64] A. B. Zемконо, Bilevel Programming: Reformulations, Regularity, and Stationarity, Ph.D. thesis (S. Dempe, advisor), Fakultät für Mathematik und Informatik, TU Bergakademie Freiberg, Freiberg, 2012.

Copyright $@$ by SIAM. Unauthorized reproduction of this article is prohibited. 